\title{
BOUNDED REMAINDER SETS UNDER TORUS EXCHANGE TRANSFORMATIONS
}

\author{
V. G. ZHURAVLEV
}

\begin{abstract}
The object of study is a certain class $\mathbb{S}$ of exchange transformations of the two-dimensional torus $\mathbb{T}^{2}$, obtained by perturbing direct products of two onedimensional interval exchange transformations. An explicit construction of bounded remainder sets under transformations in $\mathbb{S}$ is given. Sharp bounds for the deviation functions of such sets are proved and their mean values are calculated.
\end{abstract}

\section{INTRODUCTION}

0.1. Torus exchange transformations. Consider the two-dimensional torus $\mathbb{T}^{2}=$ $\mathbb{R}^{2} / \mathbb{Z}^{2}$ and a map

$$
\mathbb{T}^{2} \stackrel{\mathbb{S}}{\rightarrow} \mathbb{T}^{2}: \mathbf{x} \mapsto \mathbb{S}(\mathbf{x})=\mathbf{x}+v(\mathbf{x}) \bmod \mathbb{Z}^{2} .
$$

The map $\mathbb{S}$ is said to be translational if the set

$$
V=\left\{v(\mathbf{x}) \bmod \mathbb{Z}^{2} ; \mathbf{x} \in \mathbb{T}^{2}\right\}
$$

contains a finite number $s=\sharp V$ of distinct vectors $\bmod \mathbb{Z}^{2}$. For example, if $s=1$, then the map $\mathbb{S}=\mathbb{S}_{v}$ is the usual translation (rotation) of the torus $\mathbb{T}^{2}$ by a fixed vector $v \in \mathbb{T}^{2}$ :

$$
\mathbb{T}^{2} \stackrel{\mathbb{S}_{v}}{\longrightarrow} \mathbb{T}^{2}: \mathbf{x} \mapsto \mathbb{S}_{v}(\mathbf{x})=\mathbf{x}+v \bmod \mathbb{Z}^{2}
$$

In the general case of a translational map $\mathbb{S}$, the torus $\mathbb{T}^{2}$ can be uniquely partitioned

$$
\mathbb{T}^{2}=\mathcal{T}_{0} \sqcup \mathcal{T}_{1} \sqcup \cdots \sqcup \mathcal{T}_{s-1}
$$

into sets $\mathcal{T}_{k}=\left\{\mathbf{x} \in \mathbb{T}^{2} ; v(\mathbf{x})=v_{k}\right\}$, where $v_{0}, v_{1}, \ldots, v_{s-1}$ is the complete collection of distinct vectors in $V$. If, moreover, the images $\mathbb{S}\left(\mathcal{T}_{k}\right)$ of the sets $\mathcal{T}_{k}$ occurring in (0.1) under the map $\mathbb{S}$ again form a partition of the torus,

$$
\mathbb{T}^{2}=\mathbb{S}\left(\mathcal{T}_{0}\right) \sqcup \mathbb{S}\left(\mathcal{T}_{1}\right) \sqcup \cdots \sqcup \mathbb{S}\left(\mathcal{T}_{s-1}\right),
$$

then the map $\mathbb{S}$ is called an exchange transformation of the torus $\mathbb{T}^{2}$. Condition (0.2) can be replaced by an equivalent one: the translational map $\mathbb{S}$ must be a bijection of the torus $\mathbb{T}^{2}$.

0.2. Bounded remainder sets. For an arbitrary subset $X$ of $\mathbb{T}^{2}$, we define its distribution function

$$
\mathbf{r}_{X}\left(i, \mathbf{x}_{0}\right)=\sharp\left\{j ; \mathbb{S}^{j}\left(\mathbf{x}_{0}\right) \in X ; 0 \leq j<i\right\}
$$

to be equal to the number of points $\mathbf{x}_{j}=\mathbb{S}^{j}\left(\mathbf{x}_{0}\right)$ for $0 \leq j<i$ in the orbit $\operatorname{Orb}\left(\mathbf{x}_{0}, \mathbb{S}\right)$ that lie in the set $X$. Here $\mathbf{x}_{0} \in \mathbb{T}^{2}$ is the starting point of an orbit. The set $X$ is called

2010 Mathematics Subject Classification. Primary 05B45; Secondary 51M20.

Key words and phrases. Torus exchange transformations, bounded remainder sets on the torus, multidimensional Hecke theorem.

Supported by RFBR (grant no. 14-01-00360). 
a bounded remainder set, or a $B R$-set, if there exists a number $\mu(X)>0$ such that the deviation function

$$
\delta_{X}\left(i, \mathbf{x}_{0}\right)=\mathbf{r}_{X}\left(i, \mathbf{x}_{0}\right)-i \mu(X)
$$

is bounded:

$$
m \leq \delta_{X}\left(i, \mathbf{x}_{0}\right) \leq M
$$

for all $i=0,1,2, \ldots$, where $m$ and $M$ are finite bounds.

0.3. Main results. In the present paper we consider exchange transformations $\mathbb{S}$ of the torus $\mathbb{T}^{2}$ that are obtained by perturbing direct products of two one-dimensional exchange transformations. For such $\mathbb{S}$ the following inequalities are proved in Theorem 5.1

$$
\begin{aligned}
m-x_{0} & \leq \delta_{\mathbb{T}_{0}^{2}}\left(i, \mathbf{x}_{0}\right) \leq M-x_{0}, \\
x_{0}-M & \leq \delta_{\mathbb{T}_{1}^{2}}\left(i, \mathbf{x}_{0}\right) \leq x_{0}-m
\end{aligned}
$$

for all $i=0,1,2, \ldots$ Here we take $X \subset \mathbb{T}^{2}$ to be bounded remainder sets $\mathbb{T}_{0}^{2}$ and $\mathbb{T}_{1}^{2}$ for some partition of the torus

$$
\mathbb{T}^{2}=\mathbb{T}_{0}^{2} \sqcup \mathbb{T}_{1}^{2}
$$

given by an exchange transformations $\mathbb{S}$, the quantities $m$ and $M$ are the bounds of an explicitly defined toric development $\widehat{T} \subset \mathbb{R}^{2}$, and $x_{0}$ is the projection of the starting point $\mathbf{x}_{0} \in \mathbb{T}^{2}$ to the real line $\mathbb{R}$. If the orbit $\operatorname{Orb}\left(\mathbf{x}_{0}, \mathbb{S}\right)$ is dense on the torus $\mathbb{T}^{2}$, then the bounds for the deviations (0.5) are sharp.

Under certain additional conditions on an exchange transformation $\mathbb{S}$, Theorem 7.1 provides the mean values $\left\langle\delta_{\mathbb{T}_{0}^{2}}\left(\mathbf{x}_{0}\right)\right\rangle$ and $\left\langle\delta_{\mathbb{T}_{1}^{2}}\left(\mathbf{x}_{0}\right)\right\rangle$ for the deviations in (0.3):

$$
\left\langle\delta_{\mathbb{T}_{0}^{2}}\left(\mathbf{x}_{0}\right)\right\rangle=\int_{\widehat{T}} x d \mu(x)-x_{0}, \quad\left\langle\delta_{\mathbb{T}_{1}^{2}}\left(\mathbf{x}_{0}\right)\right\rangle=x_{0}-\int_{\widehat{T}} x d \mu(x),
$$

where $\mu(x)$ is an invariant measure on the toric development $\widehat{T}$.

0.4. History of the problem. Hecke [3] was the first to discover bounded remainder sets in 1921. Oren [6] was able to use finite unions of intervals instead.

In 1954, Szüsz [8] constructed the first family of $B R$-parallelograms. Liardet [5] discovered a possibility to reduce $B R$-sets to similar sets of smaller dimension. This was an important step, because it allowed to construct $B R$-sets of arbitrary dimension $D$. Rauzy [7] and Ferenczi [4 related the $B R$-sets to properties of the corresponding induced map - the first recurrence map or Poincaré map. The idea of Rauzy-Ferenczi was realized in [1], where inequalities of the form (0.4) were proved for sets in two-dimensional toric partitions of Rauzy.

A global approach to finding $B R$-sets was suggested by the author in 2011 in the paper [12, where instead of the sets $X$ themselves, complete partitions of the tori $\mathbb{T}^{D}=$ $X_{0} \sqcup X_{1} \sqcup \cdots \sqcup X_{s-1}$ into $B R$-sets $X_{0}, X_{1}, \ldots, X_{s-1}$. were considered. The main idea was to define a lift of the torus $\mathbb{T}^{D}$ to the covering space $\mathbb{R}^{D}$ and to consider not the torus $\mathbb{T}^{D}$ but rather its development $T^{D} \subset \mathbb{R}^{D}$. These developments $T^{D}$ were constructed in 13 by combining the stretching of unit cubes and the method of cuts in [14]. In the paper [12], a multidimensional generalization of the Hecke theorem on fractional parts distribution was obtained for the first time, and the mean values of the deviation functions (0.6) were calculated, while in [15] continuous families of $B R$-polytopes of an arbitrary dimension $D$ were constructed. In particular, in dimensions 3 and 4 they contain Fedorov's [18] and Voronoi's [10] parallelohedra. 


\section{$\S 1$. EXCHANGED SETS}

\subsection{Translational map of the line. Consider a partition}

$$
\mathbb{R}=R_{0} \sqcup R_{1}
$$

of the real line $\mathbb{R}$ into two half-closed intervals $R_{0}=[-\infty, \omega)$ and $R_{1}=[\omega,+\infty$, ), where $\omega \in \mathbb{R}$ is an arbitrary cut. Using the partition (1.1), we define a map

$$
\mathbb{R} \stackrel{S_{v}}{\longrightarrow} \mathbb{R}: S_{v}(x)=x+v(x),
$$

where $v(x)=v_{k}$ for $x \in R_{k}, k=0,1$. Here $v_{0}, v_{1}$ are some numbers in $\mathbb{R}$. The map $S_{v}$ is said to be translational, because it maps any point $x \in \mathbb{R}$ by some translation into its image $S_{v}(x)=x+v(x)$.

Denote by $I=[0,1)$ the unit left-closed interval, and let $\alpha \in I, \alpha \neq 0$. In the definitions (1.1) and (1.2), choose

$$
\omega=1-\alpha, \quad v_{0}=\alpha, \quad v_{1}=\alpha-1 .
$$

Under these assumptions, the map $S_{v}$ has the property

$$
S_{v}(\mathbb{R})=\mathbb{R},
$$

i.e., $\mathbb{R}$ is a stabilizer of the map $S_{v}$. However, if we replace $\mathbb{R}$ by an arbitrary finite interval $\mathbb{R}_{c}=\{x \in \mathbb{R} ;|x| \leq c\}$ with $c>1$, we shall have the strict inclusion

$$
S_{v}\left(\mathbb{R}_{c}\right) \subset \mathbb{R}_{c} .
$$

Therefore, $S_{v}$ is a retractive map on the intervals $\mathbb{R}_{c}$. Note that it is not a contraction map, because the distance between the images of two points may reduce and may remain the same.

We find the attractor

$$
\operatorname{Att}_{S_{v}, c}=\bigcap_{1 \leq i<+\infty} S_{v}^{i}\left(\mathbb{R}_{c}\right)
$$

of the map $S_{v}$ on the intervals $\mathbb{R}_{c}$. It is easily seen that the attractor $\operatorname{Att}_{S_{v}, c}$ does not depend on the choice of the parameter $c>1$, and

$$
\operatorname{Att}_{S_{v}, c}=I \text {. }
$$

Consider a partition

$$
T=T_{0} \sqcup T_{1}
$$

of the unit half-closed interval $I$ into two half-closed intervals $T_{0}=[0, \omega)$ and $T_{1}=[\omega, 1)$. Then the restriction of the map $S_{v}$ to a set $T \subset \mathbb{R}$ gives a bijection

$$
S_{v}: T T,
$$

on it, which is an exchange transformation of the half-closed intervals $T_{0}$ and $T_{1}$. A half-closed interval $T$ is an attracting set with the following properties: 1) after a finite number of steps, any point $x \in \mathbb{R}$ is mapped by $S_{v}$ into the half-interval $T$, and 2) then stays in it by (1.6). In this case we say that such a map is of finite type.

1.2. Exchange transformation of the unit half-closed interval. Suppose $\alpha^{\prime} \in I$, $\alpha^{\prime} \neq 0$. Choose $\omega^{\prime}=1-\alpha^{\prime}, v_{0}^{\prime}=\alpha^{\prime}, v_{1}^{\prime}=\alpha^{\prime}-1$, and let

$$
T^{\prime}=T_{0}^{\prime} \sqcup T_{1}^{\prime}
$$

be a partition of $I$ into two half-closed intervals $T_{0}^{\prime}=\left[0, \omega^{\prime}\right), T_{1}^{\prime}=\left[\omega^{\prime}, 1\right)$. Like in (1.2), we define a map

$$
T^{\prime} \stackrel{S_{v^{\prime}}}{\longrightarrow} T^{\prime}: S_{v^{\prime}}\left(x^{\prime}\right)=x^{\prime}+v^{\prime}\left(x^{\prime}\right)
$$


on $T^{\prime}$, where $v^{\prime}\left(x^{\prime}\right)=v_{k^{\prime}}^{\prime}$ for $x^{\prime} \in T_{k^{\prime}}^{\prime}, k^{\prime}=0,1$. The definitions (1.8) and (1.9) show that $S_{v^{\prime}}$ is an exchange transformation of the half-closed intervals $T_{0}^{\prime}, T_{1}^{\prime}$ from $T^{\prime}$, and therefore, $S_{v^{\prime}}$ is a bijection.

Next, consider a strip

$$
\widehat{\mathbb{R}}=\mathbb{R} \times T^{\prime}
$$

and the direct product $S_{v} \times S_{v^{\prime}}$ of the maps $S_{v}, S_{v^{\prime}}$ on it. In order to distinguish their domains, we call the real line $\mathbb{R}$ a base, and the half-closed interval $T^{\prime}$ a fiber of the strip $\widehat{\mathbb{R}}$.

1.3. Gluing. Consider a rectangle

$$
\widehat{W}=W \times T^{\prime}
$$

in the strip (1.10), where $W=\left[w_{0}, w_{1}\right)$ is some half-closed interval containing the point $\omega$. Suppose the rectangle $\widehat{W}$ is partitioned in some way:

$$
\widehat{W}=\widehat{W}_{0} \sqcup \widehat{W}_{1} \text {. }
$$

The rectangle $\widehat{W} \subset \widehat{\mathbb{R}}$ is called a patch, and the partition (1.12) is called a coloring of the patch, alluding to the fact that points in $\widehat{W}$ have two colors, 0 and 1 . The patch $\widehat{W}$ together with its partition (1.12) will play the role of perturbation for the direct product map $S_{v} \times S_{v^{\prime}}$, which we study in the next section.

1.4. Translation map $S_{\widehat{v}}$ of the strip $\widehat{\mathbb{R}}$. Now we consider the map $S_{v} \times S_{v^{\prime}}$ on the strip $\widehat{\mathbb{R}}$. We are interested not in this map itself, but rather in its perturbation by a patch $\widehat{W}$. We define a coloring of the strip $\widehat{\mathbb{R}}$ by setting

$$
\widehat{R}=\coprod_{k, k^{\prime}} \widehat{R}_{k, k^{\prime}},
$$

where

$$
\begin{aligned}
& \widehat{R}_{0,0}=\left(R_{0} \backslash W\right) \times T_{0}^{\prime} \cup\left(\widehat{W}_{0} \cap \mathbb{R} \times T_{0}^{\prime}\right), \\
& \widehat{R}_{0,1}=\left(R_{0} \backslash W\right) \times T_{1}^{\prime} \cup\left(\widehat{W}_{0} \cap \mathbb{R} \times T_{1}^{\prime}\right), \\
& \widehat{R}_{1,0}=\left(R_{1} \backslash W\right) \times T_{0}^{\prime} \cup\left(\widehat{W}_{1} \cap \mathbb{R} \times T_{0}^{\prime}\right), \\
& \widehat{R}_{1,1}=\left(R_{1} \backslash W\right) \times T_{1}^{\prime} \cup\left(\widehat{W}_{1} \cap \mathbb{R} \times T_{1}^{\prime}\right) .
\end{aligned}
$$

Using the partition (1.13), we define a map

$$
S_{\widehat{v}}(\widehat{x})=\widehat{x}+\widehat{v}(\widehat{x}),
$$

on the strip $\widehat{\mathbb{R}}$, where $\widehat{v}(\widehat{x})=\widehat{v}_{k, k^{\prime}}$ for $\widehat{x} \in \widehat{R}_{k, k^{\prime}}$. Here the translation vectors $\widehat{v}_{k, k^{\prime}}=$ $\left(v_{k}, v_{k^{\prime}}^{\prime}\right)$ have coordinates defined in (1.3) and (1.9). From the definition it follows that the strip $\widehat{\mathbb{R}}$ is closed,

$$
S_{\widehat{v}}: \widehat{\mathbb{R}} \longrightarrow \widehat{\mathbb{R}}
$$

under the map (1.14). The map $S_{\widehat{v}}$, as well as the map $S_{v}$ in (1.2), is called a translation map, and it is again a retraction. In this case the map $S_{\widehat{v}}$ retracts

$$
\bigcap_{1 \leq i<+\infty} S_{\widehat{v}}^{i}\left(\widehat{\mathbb{R}}_{c}\right) \subset \widehat{W}_{v}
$$

any rectangle $\widehat{\mathbb{R}}_{c}=\mathbb{R}_{c} \times I$ with a parameter (see (1.4)

$$
c>\max \left\{w_{1}+v_{0},\left|w_{0}+v_{1}\right|\right\}
$$


to a rectangle

$$
\widehat{W}_{v}=\left(\widehat{W}+v_{0} \cdot I\right) \cup\left(\widehat{W}+v_{1} \cdot I\right),
$$

where $\widehat{W}+v_{k} \cdot I$ denotes the Minkowski sum of two sets $\widehat{W}$ and $v_{k} \cdot I=\left\{t v_{k} ; t \in I\right\}$. This means that, after a finite number of steps, any point $\widehat{x} \in \widehat{\mathbb{R}}$ is mapped by $S_{\widehat{v}}$ to the rectangle $\widehat{W}_{v}$. The rectangle $\widehat{W}_{v}$ itself is closed under this map, $S_{\widehat{v}}: \widehat{W}_{v} \longrightarrow \widehat{W}_{v}$, but is not its stabilizer, because we have the proper inclusion $S_{\widehat{v}}\left(\widehat{W}_{v}\right) \subset \widehat{W}_{v}$.

To find a stabilizer

$$
\widehat{T}=\bigcap_{1 \leq i<+\infty} S_{\widehat{v}}^{i}\left(\widehat{\mathbb{R}}_{c}\right)
$$

in the two-dimensional case is a more challenging problem than a similar one-dimensional problem treated in Subsection 1.1. Moreover, for an arbitrary patch $\widehat{W}$, it seems impossible to give an explicit geometric description of a stabilizer $\widehat{T}$. A stabilizer $\widehat{T}$ has the simplest structure for patches $\widehat{W}$ of small width.

1.5. Stabilizer of the map $S_{\widehat{v}}$. Consider the subset

$$
\widehat{T}=S_{\widehat{v}}\left(\widehat{R}_{0}\right) \cap S_{\widehat{v}}\left(\widehat{R}_{1}\right)
$$

of the rectangle $\widehat{W}_{v}$, where

$$
\widehat{R}_{0}=\coprod_{k^{\prime}} \widehat{R}_{0, k^{\prime}}, \quad \widehat{R}_{1}=\coprod_{k^{\prime}} \widehat{R}_{1, k^{\prime}} .
$$

Note that every $\widehat{R}_{k}$ is exactly the $k$-colored subset of the strip $\widehat{\mathbb{R}}$. We carry the partition of the strip (1.13) over to the set $\widehat{T} \subset \widehat{\mathbb{R}}$ by setting

$$
\widehat{T}=\coprod_{k, k^{\prime}} \widehat{T}_{k, k^{\prime}}
$$

where the sets

$$
\widehat{T}_{k, k^{\prime}}=\widehat{R}_{k, k^{\prime}} \cap \widehat{T} \subset \widehat{T}
$$

are the $\left(k, k^{\prime}\right)$-regions of the set $\widehat{T}$.

Proposition 1.1. Assume that $S_{\widehat{v}}$ is the map in (1.14), and $\widehat{T} \subset \widehat{\mathbb{R}}$ is the subset defined in (1.20). Assume that

$$
|W|<\min \left\{\left|v_{0}\right|,\left|v_{1}\right|\right\},
$$

where $|W|$ is the length of the interval $W$ as in (1.11). Then $\widehat{T}$ is closed under the map $S_{\widehat{v}}$ :

$$
S_{\widehat{v}}: \widehat{T} \longrightarrow \widehat{T}
$$

Proof. Consider a partition

$$
\widehat{T}=\widehat{T}_{0} \sqcup \widehat{T}_{1}
$$

of the set (1.21) into two regions

$$
\widehat{T}_{0}=\coprod_{k^{\prime}} \widehat{T}_{0, k^{\prime}}, \quad \widehat{T}_{1}=\coprod_{k^{\prime}} \widehat{T}_{1, k^{\prime}},
$$

which are 0- and 1-colored, respectively. Moreover, consider another partition

$$
\widehat{T}=\widehat{T}_{l} \sqcup \widehat{T}_{c} \sqcup \widehat{T}_{r}
$$


of the set $\widehat{T}$ into regions

$$
\begin{aligned}
& \widehat{T}_{l}=\widehat{T} \cap\left(\left[w_{0}+v_{1}, w_{1}+v_{1}\right) \times T^{\prime}\right), \\
& \widehat{T}_{c}=\left[w_{1}+v_{1}, w_{0}+v_{0}\right) \times T^{\prime}, \\
& \widehat{T}_{r}=\widehat{T} \cap\left(\left[w_{0}+v_{0}, w_{1}+v_{0}\right) \times T^{\prime}\right),
\end{aligned}
$$

where the central region $\widehat{T}_{c}$ is a rectangle independent of the coloring (perturbation) (1.12) of the patch $\widehat{W}$; more precisely, the size and position of the rectangle $\widehat{T}_{c}$ are independent, although, of course, its coloring depends on (1.12)).

Suppose that $\widehat{x}$ is an arbitrary point in $\widehat{T}$; to be definite, assume that $\widehat{x}$ lies in $\widehat{T}_{0}$.

Case 1. Suppose that $\widehat{x}$ lies in the region $\widehat{T}_{l}$, see (1.26). Then $S_{\widehat{v}}(\widehat{x})=\widehat{x}+\widehat{v}_{(0, *)}$ and, therefore, $S_{\widehat{v}}(\widehat{x}) \in \widehat{T}_{c}$ by condition (1.22). Since $\widehat{T}_{c} \subset \widehat{T}$, we have $S_{\widehat{v}}(\widehat{x}) \in \widehat{T}$, whence

$$
S_{\widehat{v}}\left(\widehat{T}_{l}\right) \subset \widehat{T}
$$

and (1.23) is proved in this case.

Case 2. Now suppose that $\widehat{x}$ lies in the region $\widehat{T}_{c}$. By assumption, we have $\widehat{x} \in \widehat{T}_{0}$, and it clearly follows that $S_{\widehat{v}}(\widehat{x}) \in S_{\widehat{v}}\left(\widehat{T}_{0}\right)$. But by (1.20) we have the inclusion $S_{\widehat{v}}\left(\widehat{T}_{0}\right) \subset$ $S_{\widehat{v}}\left(\widehat{R}_{0}\right)$, so that

$$
S_{\widehat{v}}(\widehat{x}) \in S_{\widehat{v}}\left(\widehat{R}_{0}\right) .
$$

Now, by the definition (1.20), in order to prove (1.23), we need to check that

$$
S_{\widehat{v}}(\widehat{x}) \in S_{\widehat{v}}\left(\widehat{R}_{1}\right) \text {. }
$$

Consider the strip

$$
\widehat{R}\left(w_{1}+v_{1}\right)=\left\{\widehat{x}=\left(x, x^{\prime}\right) \in \widehat{R} ; x \in\left[w_{1}+v_{1},+\infty\right)\right\},
$$

infinite to the right. Note that

$$
S_{\widehat{v}}(\widehat{x}) \in \widehat{R}\left(w_{1}+v_{1}\right),
$$

because by the definition (1.27) we have $\widehat{T}_{c} \subset \widehat{R}\left(w_{1}+v_{1}\right)$, and $S_{\widehat{v}}(\widehat{x})=\widehat{x}+\widehat{v}_{(0, *)}$ is a shift to the right. Using (1.31), we obtain

$$
\widehat{R}\left(w_{1}+v_{1}\right) \subset S_{\widehat{v}}\left(\widehat{R}_{1}\right) .
$$

Applying (1.32) and (1.33), we get (1.30). Together with the relation (1.29) obtained earlier, this implies by (1.20) that $S_{\widehat{v}}(\widehat{x}) \in \widehat{T}$. Therefore we have

$$
S_{\widehat{v}}\left(\widehat{T}_{c}\right) \subset \widehat{T},
$$

and the closedness property (1.23) in the second case is proved.

Now (1.28) and (1.34) imply

$$
S_{\widehat{v}}\left(\widehat{T}_{0}\right) \subset \widehat{T} .
$$

The case where $\widehat{x}$ lies in $\widehat{T}_{1}$ (see $(1.25)$ ) is completely symmetric to that analyzed above. Hence, the map $S_{\widehat{v}}$ is closed in the sense of (1.23) for the entire set $\widehat{T}$.

Remark 1.1. The proof of Proposition 1.1 implies that

$$
\widehat{T}=S_{\widehat{v}}(\widehat{T}), \quad \widehat{T}=S_{\widehat{v}}\left(\widehat{W}_{v}\right) .
$$

The first relation in (1.35) shows that the set $\widehat{T}$ is a stabilizer under the translation map $S_{\widehat{v}}$, while the second relation and (1.16) imply that the map $S_{\widehat{v}}$ is of finite type. The simplest stabilizer $\widehat{T}$ is shown in Figure 1.1. Here the patch $\widehat{W}=\left[w_{0}, w_{1}\right) \times[0,1)$ is divided horizontally, $\widehat{W}=\widehat{W}_{0} \sqcup \widehat{W}_{1}$, into two strips $\widehat{W}_{0}$ and $\widehat{W}_{1}$ of the same height. The mapping $S_{\widehat{v}}$ on the set $\widehat{T}$ reduces to the exchange of four connected polygonal regions 


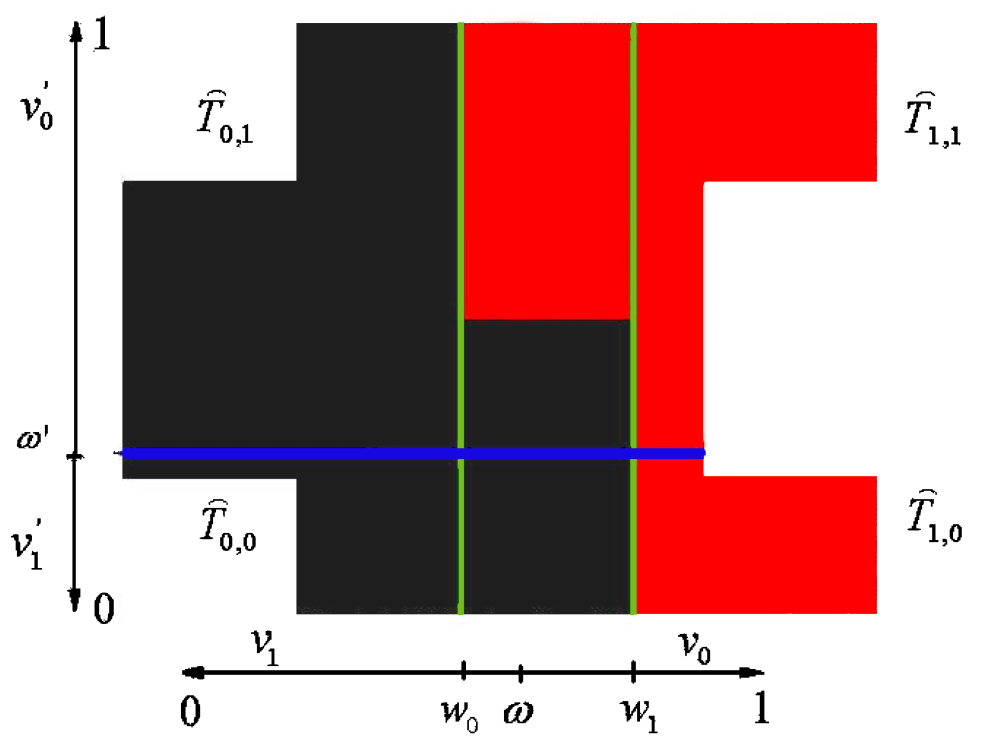

Figure 1.1. The stabilizer $\widehat{T}$ of the translational map $S_{\widehat{v}}$.

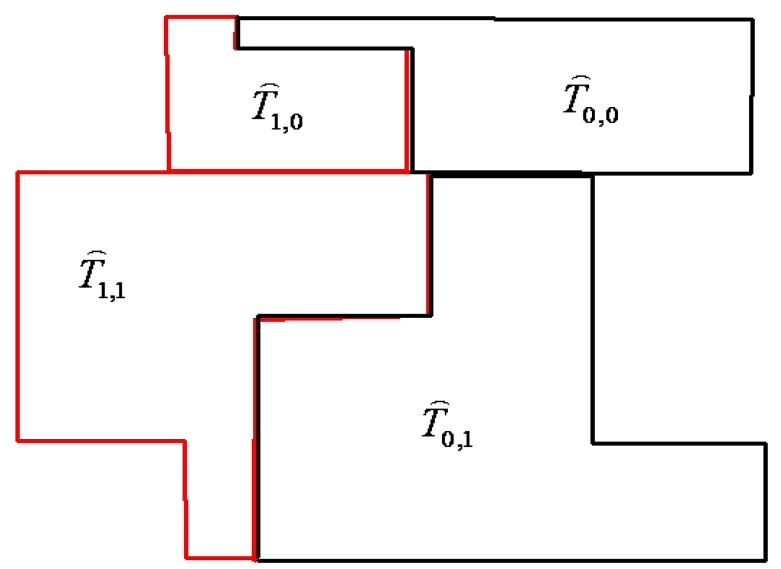

Figure 1.2. Exchange of the stabilizer $\widehat{T}$ under the map $S_{\widehat{v}}$.

$\widehat{R}_{k, k^{\prime}}$ defined in (1.13): the regions $\widehat{R}_{0,0}$ and $\widehat{R}_{0,1}$ on the left, divided by the horizontal line $\omega^{\prime}$, and similarly, the regions $\widehat{R}_{1,0}, \widehat{R}_{1,1}$ on the right of a different color. Figure 1.2 shows the position of the regions $\widehat{R}_{k, k^{\prime}}$ after the exchange. Together they form the original set $\widehat{T}$ again. From this it follows directly that the stabilizer $\widehat{T}=S_{\widehat{v}}(\widehat{T})$ is closed under the $\operatorname{map} S_{\widehat{v}}$.

Remark 1.2. In the proof of (1.37) we used the restriction (1.22) of Proposition 1.1 on the width of the strip $\widehat{W}$. If we relax (1.22), i.e., if we allow the width of the strip $\widehat{W}$ to grow, our construction (1.20) of the stabilizer $\widehat{T}$ works no longer.

For example, Figure 1.3 shows the perturbed stabilizer $\widehat{T}$ with the same parameters as in Figure 1.1, but under the condition $|W|>\min \left\{\left|v_{0}\right|,\left|v_{1}\right|\right\}$. 


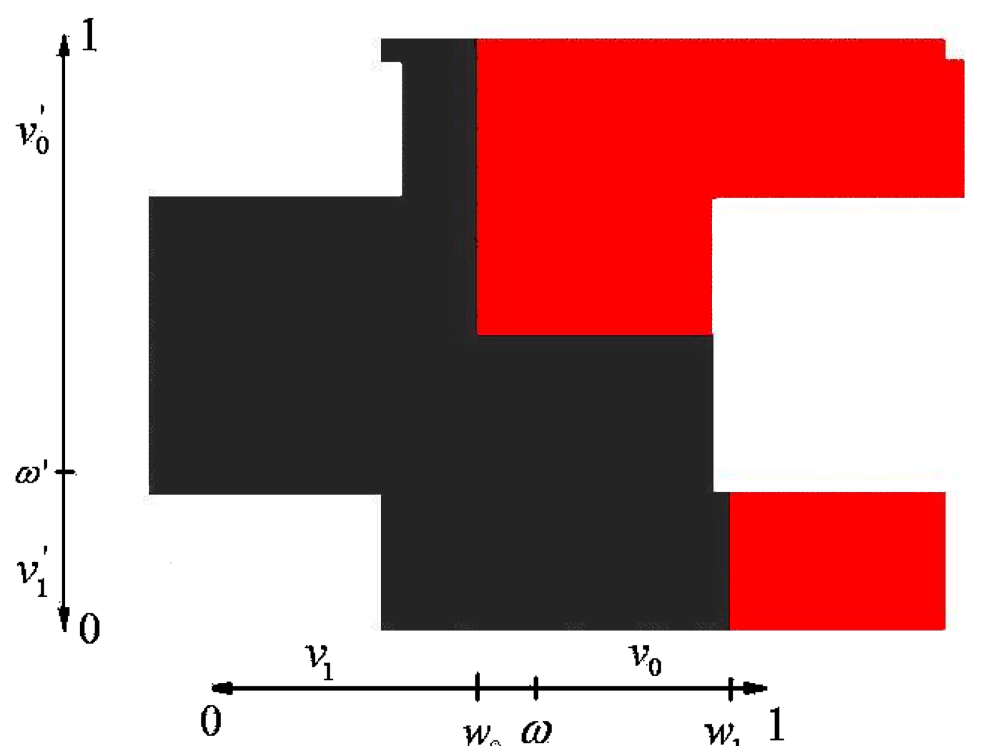

FiguRE 1.3. The perturbed stabilizer $\widehat{T}$ of the map $S_{\widehat{v}}$.

1.6. Tori. Let $L=\mathbb{Z}\left[l_{1}, l_{2}\right]$ be a complete lattice in $\mathbb{R}^{2}$, i.e., a $\mathbb{Z}$-module with a basis $l_{1}, l_{2} \in \mathbb{R}^{2}$ that is linearly independent over $\mathbb{R}$. The quotient space $\mathbb{R}^{2} / L$ is called the torus $\mathbb{T}_{L}^{2}$. A development $T_{L}^{2}$ of the torus $\mathbb{T}_{L}^{2}$ is any subset $T_{L}^{2}$ of $\mathbb{R}^{2}$ such that the canonical map

$$
T_{L}^{2} \stackrel{\bmod L}{\longrightarrow} \mathbb{T}_{L}^{2}: \widehat{x} \mapsto \widehat{x} \bmod L
$$

is bijective. A more geometric definition of the development $T_{L}^{2}$ relates it to the fundamental domain $T_{L}^{2} \subset \mathbb{R}^{2}$ of the translation group $L$ of the plane $\mathbb{R}^{2}: \widehat{x} \rightarrow \widehat{x}+l$ for $l \in L$. By definition, a fundamental domain $T_{L}^{2}$ is a subset in $\mathbb{R}^{2}$ that splits the plane $\mathbb{R}^{2}$,

$$
\mathbb{R}^{2}=\coprod_{l \in L} T_{L}^{2}[l]
$$

Here $T_{L}^{2}[l]=T_{L}^{2}+l$ denotes the translation of the domain $T_{L}^{2}$ by a vector $l$, and $T_{L}^{2}\left[l_{1}\right] \cap$ $T_{L}^{2}\left[l_{2}\right]=\varnothing$ for all $l_{1} \neq l_{2}$.

A lattice $L=\mathbb{Z}\left[l_{1}, l_{2}\right]$ is said to be square if $l_{1}=e_{1}=(1,0), l_{2}=e_{2}=(0,1)$ is the standard basis. In this case we use a shorter notation $\mathbb{Z}^{2}$ for the lattice $\mathbb{Z}\left[e_{1}, e_{2}\right]$, and, respectively, $\mathbb{T}^{2}=\mathbb{T}_{\mathbb{Z}^{2}}^{2}$ and $T^{2}=T_{\mathbb{Z}^{2}}^{2}$ for the torus and its development.

Lemma 1.1. Suppose that $X^{2}$ is some subset of the torus development $T_{L}^{2}$, and let $X^{2}[l]$ be a translation of this set by an arbitrary vector $l$ of the lattice $L$. Then the deformed subset

$$
T^{*}=\left(T_{L}^{2} \backslash X^{2}\right) \cup X^{2}[l]
$$

is again a torus development $\mathbb{T}_{L}^{2}$ with respect to the same lattice $L$.

Proof. This follows from the relation $T^{*} / L=T_{L}^{2} / L$ and the definition of the development (1.36). 


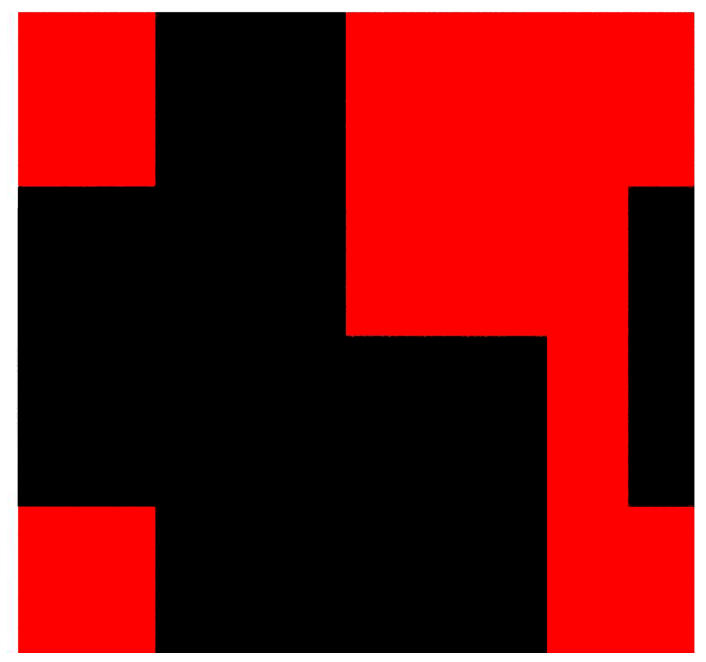

Figure 1.4. The quotient $\mathbb{T}^{2}$ of the torus development $\widehat{T} \bmod \mathbb{Z}^{2}$.

\subsection{Stabilizer $\widehat{T}$ and torus development.}

Theorem 1.1. If a patch $\widehat{W}$ satisfies condition (1.22), then the set $\widehat{T}$ defined in (1.20) is a development

$$
\widehat{T}=T^{2}
$$

of the torus $\mathbb{T}^{2}$ with respect to the square lattice $L=\mathbb{Z}^{2}$.

Proof. First of all, by the definition (1.3) of the map $S_{v}$ we can write the relation

$$
S_{v}\left(x_{0}\right)=S_{v}\left(x_{1}\right)+1
$$

linking the $S_{v}$-image of a 0 -colored point $x=x_{0}$ and that of the same point $x$ should it be 1-colored: $x=x_{1}$. Combined with the definitions (1.20), (1.27) of the set $\widehat{T}$ and its subset $\widehat{T}_{l} \subset \widehat{T}$, formula (1.38) implies that the rearranged set

$$
\widehat{T}^{*}=\left(\widehat{T} \backslash \widehat{T}_{l}\right) \cup \widehat{T}_{l}\left[e_{1}\right]
$$

is the rectangle

$$
\widehat{T}^{*}=\left[w_{1}+v_{1}, w_{1}+v_{0}\right) \times T^{\prime}=\left[w_{1}+v_{1}, w_{1}+v_{0}\right) \times[0,1),
$$

which is the unit square, because by (1.3) we have $\left(w_{1}+v_{0}\right)-\left(w_{1}+v_{1}\right)=1$. By (1.36), it is a development

$$
\widehat{T}^{*}=T^{2}
$$

of the torus $\mathbb{T}^{2}$ with respect to the square lattice $\mathbb{Z}^{2}$. Now Lemma 1.1 and relations (1.39), (1.40) imply Theorem 1.1.

Let us illustrate Theorem 1.1 by using the stabilizer $\widehat{T}$ shown in Figure 1.1. The stabilizer $\widehat{T}$ is a development of the torus $T^{2}$, which can easily be seen if we consider the quotient of $\widehat{T}$ by the square lattice $\mathbb{Z}^{2}$. As a result, we obtain the torus $\mathbb{T}^{2}$ partitioned into two sets (see Figure 1.4). But if we take the quotient of the perturbed stabilizer $\widehat{T}$ shown in Figure 1.3, we again get the torus $\mathbb{T}^{2}$, but partitioned into two slightly different subsets (see Figure 1.5). 


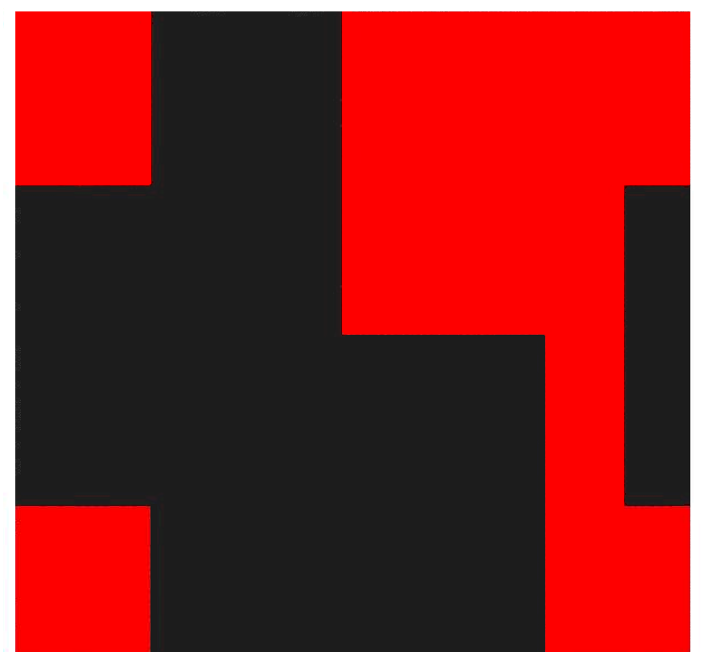

Figure 1.5. The quotient $\mathbb{T}^{2}$ of the perturbed torus development $\widehat{T} \bmod \mathbb{Z}^{2}$.

\section{$\S 2$. Fiber With arbitrary EXChange of Intervals}

In this and the next section we extend the above constructions of a torus development $\widehat{T}$. Specifically, we consider the following questions. How much can we modify the map $S_{\widehat{v}}$ defined in (1.14), if we want to

1) preserve the construction (1.20) of the stabilizer $\widehat{T}$ of the map $S_{\widehat{v}}$;

2) preserve not only the construction (1.20), but also the stabilizer itself?

2.1. Exchange of the fiber $T^{\prime}$. Leaving the base $\mathbb{R}$ and its map $S_{v}$ as in (1.2), (1.3) at rest, we consider the fiber $T^{\prime}$ and its exchange transformation $S_{v^{\prime}}$, see (1.8) and (1.9). Note that the definitions in (1.15) and (1.20) of the map $S_{\widehat{v}}$ and of the set $\widehat{T}$ will remain the same if, instead of an exchange transformation of the two half-closed intervals of a partition $T^{\prime}=T_{0}^{\prime} \sqcup T_{1}^{\prime}$, we use an exchange transformation of an arbitrary number of adjacent half-closed intervals $T_{k^{\prime}}^{\prime}=\left[\omega_{k^{\prime}}^{\prime}, \omega_{k^{\prime}+1}^{\prime}\right)$ of a partition

$$
T^{\prime}=T_{0}^{\prime} \sqcup T_{1}^{\prime} \sqcup \cdots \sqcup T_{\mathbf{k}^{\prime}}^{\prime}
$$

of the fiber $T^{\prime}=[0,1)$. Define the exchange transformation $S_{v^{\prime}}$ of the interval $T^{\prime}$ by

$$
T^{\prime} \stackrel{S_{v^{\prime}}}{\longrightarrow} T^{\prime}: S_{v^{\prime}}\left(x^{\prime}\right)=x^{\prime}+v^{\prime}\left(x^{\prime}\right)
$$

where $v^{\prime}\left(x^{\prime}\right)=v_{k^{\prime}}^{\prime}$ for $x^{\prime} \in T_{k^{\prime}}^{\prime}, k^{\prime}=0,1, \ldots, \mathbf{k}^{\prime}$. Here the translations $v_{k^{\prime}}^{\prime}$ are chosen from the open interval $I^{\text {int }}=(-1,1)$ so that the map in (2.2) is bijective. Geometrically, this means that the half-closed intervals $T_{0}^{\prime}, T_{1}^{\prime}, \ldots, T_{\mathbf{k}^{\prime}}^{\prime}$ permute as prescribed, and then, after permutation, form the entire half-closed interval $T^{\prime}=[0,1)$.

2.2. Translation map $S_{\widehat{v}}$ of the strip $\widehat{\mathbb{R}}$. The strip $\widehat{\mathbb{R}}=\mathbb{R} \times T^{\prime}$, the patch $\widehat{W}=W \times T^{\prime}$ and its coloring are defined the same way as in (1.10) -(1.12). Then we modify the coloring (1.13) of the strip $\widehat{\mathbb{R}}$ in accordance with the partition (2.1):

$$
\widehat{R}=\coprod_{k, k^{\prime}} \widehat{R}_{k, k^{\prime}}
$$

where the sets

$$
\widehat{R}_{k, k^{\prime}}=\left(R_{k} \backslash W\right) \times T_{k^{\prime}}^{\prime} \cup\left(\widehat{W}_{k} \cap \mathbb{R} \times T_{k^{\prime}}^{\prime}\right)
$$


are enumerated by the indices $k=0,1$ and $k^{\prime}=0,1, \ldots, \mathbf{k}^{\prime}-1$.

Remark 2.1. Note that we use three types of colorings: 1) $k$-coloring (1.6) of the unit half-closed interval $T$; 2) $k^{\prime}$-coloring (1.8) of the fiber $T^{\prime}$; and 3) $\left(k, k^{\prime}\right)$-coloring (2.3) of the strip $\widehat{\mathbb{R}}$. Every coloring uses its own color.

Define the translation map

$$
S_{\widehat{v}}: \widehat{\mathbb{R}} \longrightarrow \widehat{\mathbb{R}}
$$

of the strip $\widehat{\mathbb{R}}$ by

$$
S_{\widehat{v}}(\widehat{x})=\widehat{x}+\widehat{v}(\widehat{x}),
$$

where $\widehat{v}(\widehat{x})=\widehat{v}_{k, k^{\prime}}$ for $\widehat{x} \in \widehat{R}_{k, k^{\prime}}$. Now the translation vectors $\widehat{v}_{k, k^{\prime}}=\left(v_{k}, v_{k^{\prime}}^{\prime}\right)$ have coordinates defined in (1.3) and (2.2). The map $S_{\widehat{v}}$ retracts any rectangle $\widehat{\mathbb{R}}_{c}=\mathbb{R}_{c} \times I$, where $c>1$, in the strip $\widehat{\mathbb{R}}$ to the rectangle $\widehat{W}_{v}$ defined in (1.18) and closed under the $\operatorname{map}(2.6), S_{\widehat{v}}: \widehat{W}_{v} \longrightarrow \widehat{W}_{v}$.

Like in (1.20), define the set

$$
\widehat{T}=S_{\widehat{v}}\left(\widehat{R}_{0}\right) \cap S_{\widehat{v}}\left(\widehat{R}_{1}\right)
$$

where

$$
\widehat{R}_{0}=\coprod_{k^{\prime}} \widehat{R}_{0, k^{\prime}}, \quad \widehat{R}_{1}=\coprod_{k^{\prime}} \widehat{R}_{1, k^{\prime}}
$$

and the sets $\widehat{R}_{k, k^{\prime}}$ are defined as in (2.4). Then we obtain the partition

$$
\widehat{\mathbb{R}}=\widehat{R}_{0} \sqcup \widehat{R}_{1}
$$

of the strip $\widehat{\mathbb{R}}$ into two sets $\widehat{R}_{0}, \widehat{R}_{1}$ that are 0 - and 1 -colored, respectively. From (1.18) and (2.7) it follows that

$$
\widehat{T} \subset \widehat{W}_{v} .
$$

The partition of the strip (2.3) gives the corresponding partition

$$
\widehat{T}=\coprod_{k, k^{\prime}} \widehat{T}_{k, k^{\prime}}
$$

of the set $\widehat{T} \subset \widehat{\mathbb{R}}$ into the regions

$$
\widehat{T}_{k, k^{\prime}}=\widehat{R}_{k, k^{\prime}} \cap \widehat{T} \subset \widehat{T}
$$

which are $\left(k, k^{\prime}\right)$-colored.

Theorem 2.1. Suppose that $S_{\widehat{v}}$ is the map (2.6), and that $\widehat{T} \subset \widehat{\mathbb{R}}$ is the subset defined in (2.7). Assume that the length of the interval $W=\left[w_{0}, w_{1}\right)$ in (1.11) satisfies the inequality

$$
|W|<\min \left\{\left|v_{0}\right|,\left|v_{1}\right|\right\} .
$$

Then the following holds true.

1. We have

$$
\widehat{T}=\bigcap_{1 \leq i<+\infty} S_{v}^{i}\left(\widehat{\mathbb{R}}_{c}\right)
$$

i.e., the set $\widehat{T}$ is a stabilizer of the map $S_{\widehat{v}}$, and therefore, it is closed under the map $S_{\widehat{v}}$ :

$$
S_{\widehat{v}}: \widehat{T} \longrightarrow \widehat{T} \text {. }
$$

2. The set $\widehat{T}$ is a development

$$
\widehat{T}=T^{2}
$$


of the torus $\mathbb{T}^{2}$ with respect to the square lattice $L=\mathbb{Z}^{2}$.

Proof. We can use the same arguments as those in Proposition 1.1 and Theorem 1.1

Remark 2.2. Theorem 2.1 is still valid if we replace the exchange transformation $S_{v^{\prime}}$ in (2.2) by the exchange transformation $S_{v^{\prime}, \varepsilon^{\prime}}$ with inversions $\varepsilon^{\prime}$, where after permuting the intervals that form $T^{\prime}$ some of them are additionally inversed with respect to their midpoints.

\section{$\S 3$. ISOMORPHISMS}

3.1. Torus exchange transformation. Consider an infinite cylinder $\widehat{\mathbf{C}}=\mathbb{R} \times \mathbb{T}^{1}$ and define a bijection between the strip $\widehat{\mathbb{R}}=\mathbb{R} \times T^{\prime}$ and that cylinder:

$$
\iota^{\prime}: \widehat{\mathbb{R}} \widehat{\sim}: \widehat{x}=\left(x, x^{\prime}\right) \mapsto\left(x, x^{\prime} \bmod \mathbb{Z}\right) .
$$

Using this bijection, we can carry the map of the strip $S_{\widehat{v}}$ in (2.6) over to the cylinder $\widehat{\mathbf{C}}$ via of the commutative diagram

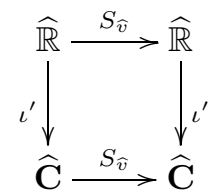

We shall preserve the old notation $S_{\widehat{v}}$ for this new map. Consider yet another quotient map of the cylinder $\widehat{\mathbf{C}}$ onto the torus,

$$
\iota: \widehat{\mathbf{C}} \longrightarrow \mathbb{T}^{2}: \widehat{x}=\left(x, x^{\prime} \bmod \mathbb{Z}\right) \mapsto \widehat{x} \bmod \mathbb{Z}^{2},
$$

where we have used the fact that $\widehat{x} \bmod \mathbb{Z}^{2}=\left(x \bmod \mathbb{Z}, x^{\prime} \bmod \mathbb{Z}\right)$. Using (2.6) and (3.3) we can carry the map $S_{\widehat{v}}$ over to the torus $\mathbb{T}^{2}$ with the help of the commutative diagram

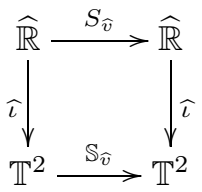

where $\widehat{\iota}=\iota \circ \iota^{\prime}$ is the composition, and the map $\mathbb{S}_{\widehat{v}}$ is given by the formula

$$
\mathbb{S}_{\widehat{v}}: \widehat{x}=\left(x, x^{\prime}\right) \mapsto\left(x+\alpha, S_{v^{\prime}}\left(x^{\prime}\right)\right) \bmod \mathbb{Z}^{2}
$$

because, by the definitions (1.2) and (1.3) of the map $S_{v}$, we have

$$
S_{v}(x) \equiv x+\alpha \bmod \mathbb{Z} .
$$

In particular, if the map $S_{v^{\prime}}$ is an exchange transformation of two intervals as in (1.9), then formula (3.5) becomes

$$
\mathbb{S}_{\widehat{v}}: \widehat{x} \mapsto \widehat{x}+\widehat{\alpha} \bmod \mathbb{Z}^{2},
$$

where $\widehat{\alpha}=\left(\alpha, \alpha^{\prime}\right)$. Hence, in this case the map

$$
\mathbb{S}_{\widehat{v}}=\mathbb{S}_{\widehat{\alpha}}
$$

is the usual shift of the torus $\mathbb{T}^{2}$ by a vector $\widehat{\alpha}$. In the general case the map $\mathbb{S}_{\widehat{v}}$ in (3.5) is an exchange transformation of the torus $\mathbb{T}^{2}$ partitioned into $2 \mathbf{k}^{\prime}$ regions. 
3.2. Coloring of the torus. Suppose that a point $\widehat{x}$ lies in the strip $\widehat{\mathbb{R}}=\widehat{R}_{0} \sqcup \widehat{R}_{1}$ with the partition as in (2.8). We introduce the notation

$$
\operatorname{col}(\widehat{x})=k
$$

for the color of the point $\widehat{x} \in \widehat{\mathbb{R}}$ if $\widehat{x} \in \widehat{R}_{k}$. By using the map $\widehat{\iota}$ in diagram (3.4), $\widehat{\iota}: \widehat{\mathbb{R}} \longrightarrow \mathbb{T}^{2}$, we can set the color for the points of the torus $\mathbb{T}^{2}$ by the formula

$$
\operatorname{col}(\widehat{\iota}(\widehat{x}))=\operatorname{col}(\widehat{x}) .
$$

Relation (3.9) does not determine the color of torus points uniquely, because the color of $\operatorname{col}(\widehat{\iota}(\widehat{x}))$ depends on the choice of its preimage $\widehat{x}$ in the strip $\widehat{\mathbb{R}}$. However, if we restrict the map (3.5) to the torus development $\widehat{T} \subset \widehat{\mathbb{R}}$, then the color $\operatorname{col}(\widehat{\iota}(\widehat{x}))$ is well defined.

In what follows we assume that the patch $\widehat{W}$ satisfies condition (2.11) of Theorem 2.1.

Lemma 3.1. 1. Assume that $\widehat{T} \subset \widehat{\mathbb{R}}$ is the torus development defined in (2.7), $\widehat{\mathbf{x}}$ is an arbitrary point of the torus $\mathbb{T}^{2}$, and $\hat{\imath}$ is the map occurring in (3.4). Then for every point $\widehat{\mathbf{x}}$ there exists a unique point $\widehat{x} \in \widehat{T}$ such that

$$
\widehat{\iota}(\widehat{x})=\widehat{\mathbf{x}} .
$$

2. Let us partition

$$
\widehat{T}=\widehat{T}_{0} \sqcup \widehat{T}_{1}
$$

the set $\widehat{T}$ into two regions

$$
\widehat{T}_{0}=\coprod_{k^{\prime}} \widehat{T}_{0, k^{\prime}}, \quad \widehat{T}_{1}=\coprod_{k^{\prime}} \widehat{T}_{1, k^{\prime}},
$$

where the sets $\widehat{T}_{k, k^{\prime}}$ are defined as in (2.10). Then the partition (3.11) gives a coloring of the set $\widehat{T}$, which in its turn induces a uniquely defined coloring

$$
\operatorname{col}(\widehat{\mathbf{x}})=\operatorname{col}(\widehat{x})
$$

of the torus $\mathbb{T}^{2}$.

Proof. The first statement follows from Theorem 2.1 and the definition (3.4) of the map $\widehat{\iota}$, because the set $\widehat{T}$ is a development of the torus $\widehat{T}=T^{2}$ with respect to the same square lattice $L=\mathbb{Z}^{2}$ as was used for the torus $\mathbb{T}^{2}$. Moreover, the development and the torus are quotient spaces of the strip $\widehat{\mathbb{R}}$.

The second statement follows from the definition (3.12) of the coloring of $\widehat{T}$ and from the definition (3.13) of the coloring of the torus $\mathbb{T}^{2}$.

Proposition 3.1. Suppose that $S_{\widehat{v}}$ is the mapping of the strip $\widehat{\mathbb{R}}$ defined in (2.6), and that $\mathbb{S}_{\widehat{v}}$ is the mapping of the torus $\mathbb{T}^{2}$ occurring in (3.4). Then the following statements hold true.

1. There is a commutative diagram

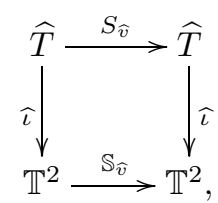

where the vertical arrows denote the bijection $\widehat{\iota}$.

2. The maps in diagram (3.14) coordinate the colors of points $\widehat{x}$ of $\widehat{T}$ and those of their images $\widehat{\mathbf{x}}=\widehat{\iota}(\widehat{x})$ on the torus $\mathbb{T}^{2}$ :

$$
\operatorname{col}\left(S_{\widehat{v}}(\widehat{x})\right)=\operatorname{col}\left(\mathbb{S}_{\widehat{v}}(\widehat{\mathbf{x}})\right) .
$$


Proof. This follows from diagram (3.4) and Lemma 1.1.

3.3. Translational map of the real line $S_{v, c}$. Consider a projection of the strip $\widehat{\mathbb{R}}$

$$
\operatorname{pr}: \widehat{\mathbb{R}} \longrightarrow \mathbb{R}: \widehat{x}=\left(x, x^{\prime}\right) \mapsto x
$$

onto the line and define the color $\operatorname{col}(x)$ of points $x$ on the line $\mathbb{R}$ by setting

$$
\operatorname{col}(x)=\operatorname{col}(\widehat{x}),
$$

where $x=\operatorname{pr}(\widehat{x})$. In general, relation (3.17) uniquely determines the color of points not on the entire line $\mathbb{R}$, but only on its part $\mathbb{R} \backslash W$, where $W=\left[w_{0}, w_{1}\right)$ is the half-closed interval occurring in (1.11). If a point $x$ lies in $W$, then the color $\operatorname{col}(x)$ depends on the choice of its preimage $\widehat{x}$ in the patch, i.e., in the rectangle $\widehat{W}=W \times T^{\prime}$ (see (1.11)).

Usually, in these situations one looks not on the entire fibered space $\widehat{\mathbb{R}}$, but rather on its sections. In our case we shall define sections by orbits

$$
\operatorname{Orb}\left(\widehat{x}_{0}, S_{\widehat{v}}\right)=\left\{\widehat{x}_{i}=S_{\widehat{v}}^{i}\left(\widehat{x}_{0}\right) ; i=0,1,2, \ldots\right\}
$$

of arbitrary starting points $\widehat{x}_{0} \in \widehat{\mathbb{R}}$ with respect to the translation map $S_{\widehat{v}}$ of the strip $\widehat{\mathbb{R}}$, defined in (2.6). We can carry the color of points from the orbit $\operatorname{Orb}\left(\widehat{x}_{0}, S_{\widehat{v}}\right)$ over to its projection

$$
x_{i}=\operatorname{pr}\left(\widehat{x}_{i}\right) \text { for } i=0,1,2, \ldots
$$

in a natural way:

$$
\operatorname{col}\left(x_{i}\right)=\operatorname{col}\left(\widehat{x}_{i}\right)
$$

Now we need to slightly modify the definitions (1.2), (1.3) of the map $S_{v}$. Let $\mathbb{R}_{c}$ be the real line $\mathbb{R}$ every point of which has $\operatorname{color} \operatorname{col}(x)=k$, where $k=0,1$. Like in (1.3), we define the map

$$
\mathbb{R}_{c} \stackrel{S_{v, c}}{\longrightarrow} \mathbb{R}_{c}: S_{v, c}(x)=x+v_{\operatorname{col}(x)},
$$

on the line $\mathbb{R}_{c}$, where $v_{0}=\alpha, v_{1}=\alpha-1$, and $\alpha \neq 0$ is some number in the unit half-closed interval $I=[0,1)$. Like $S_{v}$, the map $S_{v, c}$ is translation. Using this map, we can rewrite the projection of the orbit $\operatorname{Orb}\left(\widehat{x}_{0}, S_{\widehat{v}}\right)$ as follows:

$$
\operatorname{Orb}\left(x_{0}, S_{v, c}\right)=\left\{x_{i}=S_{v, c}^{i}\left(x_{0}\right) ; i=0,1,2, \ldots\right\},
$$

and moreover, we have the following commutative diagram:

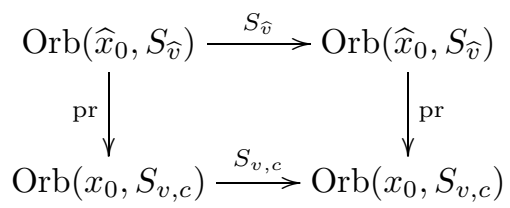

where the vertical arrows denote the projection (3.16). Here, the commutativity of the diagram (3.22), besides the usual sense, means that the colors of the points in the orbits agree:

$$
\operatorname{col}(\widehat{x})=\operatorname{col}(\operatorname{pr}(\widehat{x})), \quad \operatorname{col}\left(\operatorname{pr} \circ S_{\widehat{v}}(\widehat{x})\right)=\operatorname{col}\left(S_{\widehat{v}} \circ \operatorname{pr}(\widehat{x})\right)
$$


3.4. Toric orbits. Consider the orbits

$$
\operatorname{Orb}\left(\widehat{\mathbf{x}}_{0}, \mathbb{S}_{\widehat{v}}\right)=\left\{\widehat{\mathbf{x}}_{i}=\mathbb{S}_{\widehat{v}}^{i}\left(\widehat{\mathbf{x}}_{0}\right) ; i=0,1,2, \ldots\right\}
$$

of arbitrary starting points $\widehat{\mathbf{x}}_{0}$ on the torus $\mathbb{T}^{2}$ with respect to the exchange transformation $\mathbb{S}_{\widehat{v}}$ of the torus, see (3.5). We define the projection of the torus $\mathbb{T}^{2}$ to the line

$$
\text { pr: } \mathbb{T}^{2} \longrightarrow \mathbb{R}
$$

by using the commutative diagram

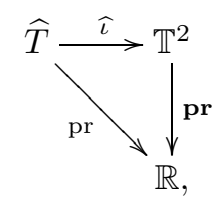

where $\widehat{T}=T^{2}$ is a development of the torus $\mathbb{T}^{2}$, the horizontal arrow denotes the bijection $\widehat{\imath}$ from Proposition 3.1, and the arrow on the left is the projection occurring in (3.16).

Proposition 3.2. For any starting point $\widehat{\mathbf{x}}_{0}$ on the torus $\mathbb{T}^{2}$, there exists a coloring of the line $\mathbb{R}_{c}=\mathbb{R}_{c, \widehat{\mathbf{x}}_{0}}$ depending on the point $\widehat{\mathbf{x}}_{0}$ and such that the following diagram is commutative:

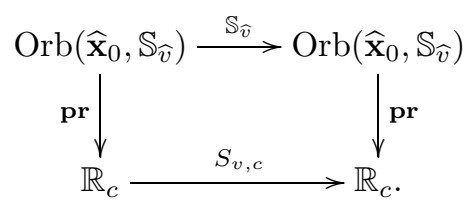

Here the vertical arrows denote the projection (3.24), and in diagram (3.25) the colors of the points $\widehat{\mathbf{x}} \in \operatorname{Orb}\left(\widehat{\mathbf{x}}_{0}, \mathbb{S}_{\widehat{v}}\right)$ and $\widehat{x}=\operatorname{pr}(\widehat{\mathbf{x}}) \in \mathbb{R}_{c}$ agree:

$$
\operatorname{col}(\widehat{\mathbf{x}})=\operatorname{col}(\widehat{x}), \quad \operatorname{col}\left(\mathbf{p r} \circ \mathbb{S}_{\widehat{v}}(\widehat{\mathbf{x}})\right)=\operatorname{col}\left(\mathbb{S}_{\widehat{v}} \circ \mathbf{p r}(\widehat{\mathbf{x}})\right),
$$

where the coloring $\operatorname{col}(\widehat{\mathbf{x}})$ of the points $\widehat{\mathbf{x}}$ on the torus $\mathbb{T}^{2}$ is defined as in (3.13).

Proof. Using the projection pr involved in (3.25), we can carry the coloring of the points $\widehat{\mathbf{x}}_{i}$ in the orbit $\operatorname{Orb}\left(\widehat{\mathbf{x}}_{0}, \mathbb{S}_{\widehat{v}}\right)$ over to their images $x_{i}=\mathbf{p r}\left(\widehat{\mathbf{x}}_{i}\right)$ on the line $\mathbb{R}$. For the rest of the points in $\mathbb{R}$ we choose arbitrary colors. Thus, we obtain a coloring of the line $\mathbb{R}_{c}$, which is not uniquely determined, but this allows us to define the map $S_{v, c}$ as in (3.20) and, therefore, to obtain the entire diagram in (3.25). Now Proposition 3.2 follows from diagram (3.22) and the agreement of colors in (3.23).

\section{$\S 4$. Distribution of POINTS ON The Line}

\subsection{Distribution functions on the line. Let}

$$
\mathbb{R}_{c}=\mathbb{R}_{c, 0} \sqcup \mathbb{R}_{c, 1}
$$

be an arbitrary coloring of the line $\mathbb{R}$ partitioned into two sets $\mathbb{R}_{c, k}, k=0,1$, that define the color $\operatorname{col}(x)=k$ of points $x \in \mathbb{R}_{c, k}$. Choose some starting point $x_{0} \in \mathbb{R}$ and consider its orbit $\operatorname{Orb}\left(x_{0}, S_{v, c}\right)$. By the definition (3.20) of the map $S_{v, c}$, any point $x_{i}$ in the orbit $\operatorname{Orb}\left(x_{0}, S_{v, c}\right)$ can be expressed as

$$
x_{i}=S_{v, c}^{i}\left(x_{0}\right)=x_{0}+\sum_{0 \leq j<i} v_{\operatorname{col}\left(x_{j}\right)},
$$

where $x_{j}=S_{v, c}^{j}\left(x_{0}\right)$ is a current point of the orbit, and the translations $v_{k}$ are given by $v_{0}=\alpha, v_{1}=\alpha-1$ for some $\alpha \neq 0$ in the unit half-closed interval $I=[0,1)$. 
For $k=0,1$, we define the distribution functions

$$
r_{k}\left(i, x_{0}\right)=\sharp\left\{j ; S_{v, c}^{j}\left(x_{0}\right) \in \mathbb{R}_{c, k} ; 0 \leq j<i\right\},
$$

i.e., $r_{k}\left(i, x_{0}\right)$ equals the number of points $x_{j}=S_{v, c}^{j}\left(x_{0}\right)$ for $0 \leq j<i$ in the orbit $\operatorname{Orb}\left(x_{0}, S_{v, c}\right)$ with $\operatorname{col}\left(x_{j}\right)=k$. Using these functions, we can rewrite (4.2) in the form

$$
x_{i}=S_{v, c}^{i}\left(x_{0}\right)=x_{0}+r_{0}\left(i, x_{0}\right) v_{0}+r_{1}\left(i, x_{0}\right) v_{1} .
$$

By the definition (4.3) and condition (4.1), there is an obvious relationship between the distribution functions $r_{0}\left(i, x_{0}\right)$ and $r_{1}\left(i, x_{0}\right)$ :

$$
r_{0}\left(i, x_{0}\right)+r_{1}\left(i, x_{0}\right)=i
$$

for all $i=0,1,2, \ldots$

4.2. Deviation functions on the line. Using (4.5), we can express the function $r_{1}\left(i, x_{0}\right)$ in terms of $r_{0}\left(i, x_{0}\right)$ and substitute it into formula (4.4). We obtain

$$
x_{i}=S_{v, c}^{i}\left(x_{0}\right)=x_{0}+r_{0}\left(i, x_{0}\right)+i v_{1},
$$

because $v_{0}-v_{1}=1$ by assumption. Consider the auxiliary functions

$$
\delta_{k}\left(i, x_{0}\right)=r_{k}\left(i, x_{0}\right)-i a_{k}
$$

for $k=0,1$ with the coefficients $a_{0}=-v_{1}$ and $a_{1}=v_{0}$. Recalling (4.2), it is convenient to express these functions in terms of one parameter

$$
a_{0}=1-\alpha, \quad a_{1}=\alpha .
$$

Let us simplify the situation greatly, assuming that the patch $\widehat{W}$ in (1.11) is empty. In this case, the partition (4.1) of the line degenerates into the partition (1.1), while the map $S_{v, c}$ (see (3.20) ) degenerates into the map $S_{v}$ as in (1.3). Assume that the starting point $x_{0}$ lies in the unit half-closed interval $I$ that is partitioned $T=T_{0} \sqcup T_{1}$ into two half-closed intervals $T_{0}=[0,1-\alpha), T_{1}=[1-\alpha, 1)$ (see (1.6)). As was said in (1.7), the map $S_{v}$ is an exchange transformation of the half-closed intervals $T_{0}$ and $T_{1}$; therefore, our map $S_{v, c}=S_{v}$ is isomorphic to the rotation of the unit circle $\mathbb{T}^{1}$ by angle $\alpha$. Suppose $\alpha$ is irrational. In this case, the points of the orbit $\operatorname{Orb}\left(x_{0}, S_{v, c}\right)$ (see (3.21) ) are uniformly distributed in the unit half-closed interval $T=T_{0} \sqcup T_{1}$. Note that the half-closed intervals $T_{0}$ and $T_{1}$ have lengths

$$
\left|T_{0}\right|=1-\alpha, \quad\left|T_{1}\right|=\alpha,
$$

respectively. By uniformity, the distribution functions in (4.3) satisfy the asymptotic relations

$$
r_{0}\left(i, x_{0}\right) \sim(1-\alpha) i, \quad r_{1}\left(i, x_{0}\right) \sim \alpha i
$$

as $i \rightarrow+\infty$. Now, comparing (4.8) and (4.9), we observe that the asymptotic formulas in (4.10) illuminate the meaning of the functions $\delta_{k}\left(i, x_{0}\right)$ defined in (4.7), namely, $\delta_{k}\left(i, x_{0}\right)$ is equal to the deviation of the number $r_{k}\left(i, x_{0}\right)$ of hits of points of the orbit $\operatorname{Orb}\left(x_{0}, S_{v, c}\right)$ into the half-closed interval $T_{k}$ from the expected average number of hits $i a_{k}$, where the coefficient $a_{k}=\left|T_{k}\right|$ is the length of the half-closed interval $T_{k}$.

Now we proceed to the case of the general coloring of the line (4.1).

Proposition 4.1. The deviation functions $\delta_{k}\left(i, x_{0}\right), k=0,1$, defined in (4.7) satisfy

$$
\delta_{0}\left(i, x_{0}\right)=x_{i}-x_{0}, \quad \delta_{1}\left(i, x_{0}\right)=x_{0}-x_{i},
$$

where $x_{i}=S_{v, c}^{i}\left(x_{0}\right)$ is a current point on the orbit $\operatorname{Orb}\left(x_{0}, S_{v, c}\right)$ indexed by $i=0,1,2, \ldots$

Proposition 4.1 implies the next claim. 
Corollary 4.1. For the deviation functions $\delta_{0}\left(i, x_{0}\right)$ and $\delta_{1}\left(i, x_{0}\right)$ we have

$$
\delta_{0}\left(i, x_{0}\right)+\delta_{1}\left(i, x_{0}\right)=0
$$

for all $i=0,1,2, \ldots$

Proof of Proposition 4.1. Substituting the expression (4.7) for the deviation function $\delta_{0}\left(i, x_{0}\right)$ in (4.6), we obtain the first identity in (4.11). The second can be proved in the same way if, using (4.5), we express the distribution function $r_{0}\left(i, x_{0}\right)$ in terms of $r_{1}\left(i, x_{0}\right)$ and substitute the result in (4.4).

\section{$\S 5$. Distribution of points on the torus}

5.1. Distribution and deviation functions on the torus. In Lemma 3.1 we defined a coloring $\operatorname{col}(\widehat{\mathbf{x}})$ of the points on the torus $\mathbb{T}^{2}$. Using it, we can partition the torus

$$
\mathbb{T}^{2}=\mathbb{T}_{0}^{2} \sqcup \mathbb{T}_{1}^{2}
$$

into sets $\mathbb{T}_{k}^{2}$ of points having the same color $\operatorname{col}(\widehat{\mathbf{x}})=k$. As in (4.3), for the exchange transformation $\mathbb{S}_{\widehat{v}}$ of the torus $\mathbb{T}^{2}$, defined in (3.5), we introduce the distribution functions

$$
\mathbf{r}_{k}\left(i, \widehat{\mathbf{x}}_{0}\right)=\sharp\left\{j ; \mathbb{S}_{\widehat{v}}^{j}\left(\widehat{\mathbf{x}}_{0}\right) \in \mathbb{T}_{k}^{2} ; 0 \leq j<i\right\}
$$

as the number of points $\widehat{\mathbf{x}}_{j}=\mathbb{S}_{\widehat{v}}^{j}\left(\widehat{\mathbf{x}}_{0}\right)$ for $0 \leq j<i$ that belong to the orbit $\operatorname{Orb}\left(\widehat{\mathbf{x}}_{0}, \mathbb{S}_{\widehat{v}}\right)$ and have the same color $\operatorname{col}(\widehat{\mathbf{x}})=k$, where $k=0,1$. The corresponding deviation functions on the torus $\mathbb{T}^{2}$ are (cf. (4.7))

$$
\delta_{k}\left(i, \widehat{\mathbf{x}}_{0}\right)=\mathbf{r}_{k}\left(i, \widehat{\mathbf{x}}_{0}\right)-i a_{k}
$$

with coefficients $a_{0}=1-\alpha$ and $a_{1}=\alpha$.

\subsection{The main result.}

Theorem 5.1. Let $\widehat{\mathbf{x}}_{0}$ be an arbitrary starting point on the torus $\mathbb{T}^{2}$, let $\mathbb{S}_{\widehat{v}}$ be the exchange transformation (3.5), and assume the conditions of Theorem 2.1. Then the following statements are true.

1. For the deviation functions $\delta_{k}\left(i, \widehat{\mathbf{x}}_{0}\right), k=0,1$, defined in (5.3) we have

$$
\delta_{0}\left(i, \widehat{\mathbf{x}}_{0}\right)=x_{i}-x_{0}, \quad \delta_{1}\left(i, \widehat{\mathbf{x}}_{0}\right)=x_{0}-x_{i},
$$

where

$$
x_{i}=\operatorname{pr}\left(\widehat{\mathbf{x}}_{i}\right) \text { for } \widehat{\mathbf{x}}_{i}=\mathbb{S}_{\widehat{v}}^{i}\left(\widehat{\mathbf{x}}_{0}\right),
$$

which is a current point in the orbit $\operatorname{Orb}\left(\widehat{\mathbf{x}}_{0}, \mathbb{S}_{\widehat{v}}\right)$ enumerated by $i=0,1,2, \ldots$

2. The deviations $\delta_{k}\left(i, \widehat{\mathbf{x}}_{0}\right)$ satisfy

$$
m(\widehat{T})-x_{0} \leq \delta_{0}\left(i, \widehat{\mathbf{x}}_{0}\right) \leq M(\widehat{T})-x_{0}, \quad x_{0}-M(\widehat{T}) \leq \delta_{1}\left(i, \widehat{\mathbf{x}}_{0}\right) \leq x_{0}-m(\widehat{T})
$$

for all $i=0,1,2, \ldots$ Here $m(\widehat{T})$ and $M(\widehat{T})$ stand for the boundary values

$$
m(\widehat{T})=\inf _{\widehat{x} \in \widehat{T}} \operatorname{pr}(\widehat{x}), \quad M(\widehat{T})=\sup _{\widehat{x} \in \widehat{T}} \operatorname{pr}(\widehat{x}),
$$

where $\widehat{T} \subset \mathbb{R}^{2}$ is the development of the torus (2.7), and pr is the projection (3.16).

Proof. 1. Using Proposition 3.2, we obtain

$$
\mathbf{r}_{k}\left(i, \widehat{\mathbf{x}}_{0}\right)=r_{k}\left(i, x_{0}\right)
$$

From (5.8) and the definitions (4.7), (5.3) we deduce that the following distribution functions are equal:

$$
\delta_{k}\left(i, \widehat{\mathbf{x}}_{0}\right)=\delta_{k}\left(i, x_{0}\right) .
$$

Now (5.9) and Proposition 4.1 imply (5.4). 
2. Consider the orbit $\operatorname{Orb}\left(\widehat{x}_{0}, S_{\widehat{v}}\right)$ defined in (3.18) for a starting point $\widehat{x}_{0}$ in $\widehat{T}$ for which $\widehat{\iota}\left(\widehat{x}_{0}\right)=\widehat{\mathbf{x}}_{0}$, where $\widehat{\iota}$ is the bijection occurring in (3.14). Since the torus development $\widehat{T}$ is closed under the map $S_{\widehat{v}}$ (see (2.13), we have $\operatorname{Orb}\left(\widehat{x}_{0}, S_{\widehat{v}}\right) \subset \widehat{T}$. Thus, by (5.4) and Proposition 4.1, the deviation functions $\delta_{k}\left(i, \widehat{x}_{0}\right)=r_{k}\left(i, \widehat{x}_{0}\right)-i a_{k}$ for the distribution of points $\widehat{x}_{j}=S_{\widehat{v}}^{j}\left(\widehat{x}_{0}\right)$ of the orbit $\operatorname{Orb}\left(\widehat{x}_{0}, S_{\widehat{v}}\right)$ in the regions $\widehat{T}_{k}$ of the partition $\widehat{T}=\widehat{T}_{0} \sqcup \widehat{T}_{1}$ (see (3.11) ) satisfy

$$
m(\widehat{T})-x_{0} \leq \delta_{0}\left(i, \widehat{x}_{0}\right) \leq M(\widehat{T})-x_{0}, \quad x_{0}-M(\widehat{T}) \leq \delta_{1}\left(i, \widehat{x}_{0}\right) \leq x_{0}-m(\widehat{T}),
$$

which implies the desired inequalities (5.6).

5.3. Saturated patch colorings. For arbitrary colorings (1.12) of the patch $\widehat{W}=$ $\widehat{W}_{0} \sqcup \widehat{W}_{1}$ and arbitrary exchange transformations $\mathbb{S}_{\widehat{v}}$ of the torus $\mathbb{T}^{2}$, inequalities (5.6), which we have proved earlier for the deviations $\delta_{k}\left(i, \widehat{\mathbf{x}}_{0}\right), k=0,1$, are not sharp. Now we formulate several conditions sufficient for the sharpness of inequalities (5.6).

A coloring $\widehat{W}=\widehat{W}_{0} \sqcup \widehat{W}_{1}$ of the patch $\widehat{W}$ in (1.12) is said to be saturated if for any $k=0,1$, any $\widehat{x}$ in the region $\widehat{W}_{k}$, and any its neighborhood $b(\widehat{x}, \varepsilon)$ of radius $\varepsilon>0$ there exists a neighborhood $b\left(\widehat{x}^{\prime}, \varepsilon^{\prime}\right)$ of radius $\varepsilon^{\prime}>0$ such that

$$
b\left(\widehat{x}^{\prime}, \varepsilon^{\prime}\right) \subset b(\widehat{x}, \varepsilon) \cap \widehat{W}_{k},
$$

i.e., all points $\widehat{y}$ in $b\left(\widehat{x}^{\prime}, \varepsilon^{\prime}\right)$ have the same color, and this color coincides with the color of $\widehat{x}, \operatorname{col}(\widehat{y})=k$.

Proposition 5.1. Suppose that a coloring $\widehat{W}=\widehat{W}_{0} \sqcup \widehat{W}_{1}$ is saturated, and that the orbit $\operatorname{Orb}\left(\widehat{\mathbf{x}}_{0}, \mathbb{S}_{\widehat{v}}\right)$ is dense in the torus $\mathbb{T}^{2}$. Then inequalities (5.6) for the deviations $\delta_{k}\left(i, \widehat{\mathbf{x}}_{0}\right)$, $k=0,1$, are sharp.

Proof. Since the coloring of the patch $\widehat{W}$ is saturated (see (5.10), the construction (2.7) of the torus development $\widehat{T}$ implies that for an arbitrary point $\widehat{x}$ in $\widehat{T}$ and any $\varepsilon>0$ there is a neighborhood

$$
b\left(\widehat{x}^{\prime}, \varepsilon^{\prime}\right) \subset \widehat{T} \cap b(\widehat{x}, \varepsilon)
$$

of radius $\varepsilon^{\prime}>0$ all points $\widehat{y}$ of which have the same $\operatorname{color}, \operatorname{col}(\widehat{y})=\operatorname{col}(\widehat{x})$. Furthermore, again, from the construction of $\widehat{T}$ together with Theorem 2.1 and the density of the orbit $\operatorname{Orb}\left(\widehat{\mathbf{x}}_{0}, \mathbb{S}_{\widehat{v}}\right)$ in the torus $\mathbb{T}^{2}$ it follows that there exists a point $\widehat{x}_{i}=\widehat{\iota}\left(\widehat{\mathbf{x}}_{i}\right)$ in $\widehat{T}$ (where $\widehat{\mathbf{x}}_{i}$ is some point of the orbit $\operatorname{Orb}\left(\widehat{\mathbf{x}}_{0}, \mathbb{S}_{\widehat{v}}\right)$ and $\widehat{\iota}$ is the bijection in (3.14)) such that $\widehat{x}_{i} \in b\left(\widehat{x}^{\prime}, \varepsilon^{\prime}\right)$. Therefore, this point has the color

$$
\operatorname{col}\left(\widehat{x}_{i}\right)=\operatorname{col}(\widehat{x})
$$

The definition (5.7) of $M(\widehat{T})$ shows that for any $\varepsilon>0$ there exists a point $\widehat{x} \in \widehat{T}$ such that $\operatorname{pr}(\widehat{x})>M(\widehat{T})-\varepsilon$. Together with (5.11), this implies the inequality

$$
\operatorname{pr}\left(\widehat{x}_{i}\right)>M(\widehat{T})-2 \varepsilon .
$$

Recalling (5.4), we get the inequality $\delta_{0}\left(i, \widehat{\mathbf{x}}_{0}\right)>M(\widehat{T})-2 \varepsilon$ for the deviation $\delta_{0}\left(i, \widehat{\mathbf{x}}_{0}\right)$ with the same number $i$ as in (5.12). Since $\varepsilon>0$ is arbitrary, we see that the inequality $\delta_{0}\left(i, \widehat{\mathbf{x}}_{0}\right) \leq M(\widehat{T})-x_{0}$ in (5.6) is sharp.

The sharpness of the remaining inequalities in (5.6) can be proved similarly. 
5.4. Deviations for torus shifts. Suppose that the exchange transformation $\mathbb{S}_{\widehat{v}}=\mathbb{S}_{\widehat{\alpha}}$ is the shift

$$
\mathbb{T}^{2} \stackrel{\mathbb{S}_{\widehat{\alpha}}}{\longrightarrow} \mathbb{T}^{2}: \widehat{x} \mapsto \widehat{x}+\widehat{\alpha} \bmod \mathbb{Z}^{2}
$$

of the torus $\mathbb{T}^{2}$ by a vector $\widehat{\alpha}=\left(\alpha, \alpha^{\prime}\right)$. Then for any starting point $\mathbf{x}_{0} \in \mathbb{T}^{2}$ the structure of the orbit $\operatorname{Orb}\left(\widehat{\mathbf{x}}_{0}, \mathbb{S}_{\widehat{\alpha}}\right)$ for the shift (5.13) can be described completely in terms of the rank of the vector $\widehat{\alpha}$.

We define the rank of a vector $\widehat{\alpha}$ over the ring $\mathbb{Z}$ of integers as

$$
\operatorname{rank}_{\mathbb{Z}}(\widehat{\alpha})=\operatorname{rank}_{\mathbb{Z}} M(\widehat{\alpha})-1,
$$

where $M(\widehat{\alpha})=\mathbb{Z}\left[1, \alpha, \alpha^{\prime}\right] \subset \mathbb{R}$ denotes the module over $\mathbb{Z}$ generated by $1, \alpha, \alpha^{\prime}$. The $\operatorname{rank} \operatorname{rank}_{\mathbb{Z}} \mathbb{Z}\left[1, \alpha, \alpha^{\prime}\right]$ is the dimension of this module over $\mathbb{Z}$. Formula (5.14) implies the inequalities

$$
0 \leq \operatorname{rank}_{\mathbb{Z}}(\widehat{\alpha}) \leq 2 .
$$

A vector $\widehat{\alpha}$ is said to be irrational if $\operatorname{rank}_{\mathbb{Z}}(\widehat{\alpha})>0$. If, moreover, the rank is maximal, $\operatorname{rank}_{\mathbb{Z}}(\widehat{\alpha})=2$, we say that this vector is maximally irrational. In the remaining case where $\operatorname{rank}_{\mathbb{Z}}(\widehat{\alpha})=0$ we say that the vector is rational. This is equivalent to the condition that the vector $\widehat{\alpha}=\left(\alpha, \alpha^{\prime}\right)$ has rational coordinates $\alpha, \alpha^{\prime} \in \mathbb{Q}$.

For a vector $\widehat{\alpha}$, the shift $\mathbb{S}_{\widehat{\alpha}}$ of the torus (5.13) is named accordingly.

We denote by $\overline{\operatorname{Orb}}\left(\widehat{\mathbf{x}}_{0}, \mathbb{S}_{\widehat{\alpha}}\right)$ the closure of the orbit $\operatorname{Orb}\left(\widehat{\mathbf{x}}_{0}, \mathbb{S}_{\widehat{\alpha}}\right)$. Then we have the formula

$$
\operatorname{dim} \overline{\operatorname{Orb}}\left(\widehat{\mathbf{x}}_{0}, \mathbb{S}_{\widehat{\alpha}}\right)=\operatorname{rank}_{\mathbb{Z}}(\widehat{\alpha})
$$

for the dimension of the closure of the orbit $\overline{\mathrm{Orb}}\left(\widehat{\mathbf{x}}_{0}, \mathbb{S}_{\widehat{\alpha}}\right)$ (with respect to the maximally irrational shifts of the torus $\mathbb{T}^{D}$ of arbitrary dimension $D$, which are usually said to be minimal, see [9], and also [16] for the remaining cases).

We can use (5.15) for maximally irrational torus shifts $\widehat{\alpha}$ to restate Proposition 5.1 as follows.

Proposition 5.2. Suppose that an exchange transformation $\mathbb{S}_{\widehat{v}}=\mathbb{S}_{\widehat{\alpha}}$ is a maximally irrational shift of the torus $\mathbb{T}^{2}$, and assume that the coloring $\widehat{W}=\widehat{W}_{0} \sqcup \widehat{W}_{1}$ of the patch (1.12) is saturated. Then inequalities (5.6) for the deviations $\delta_{k}\left(i, \widehat{\mathbf{x}}_{0}\right), k=0,1$, are sharp.

Proof. By assumption, we have $\operatorname{rank}_{\mathbb{Z}}(\widehat{\alpha})=2$. Therefore, by (5.15), the closure of the orbit $\overline{\operatorname{Orb}}\left(\widehat{\mathbf{x}}_{0}, \mathbb{S}_{\widehat{\alpha}}\right) \subset \mathbb{T}^{2}$ has dimension $\operatorname{dim} \overline{\operatorname{Orb}}\left(\widehat{\mathbf{x}}_{0}, \mathbb{S}_{\widehat{\alpha}}\right)$ equal to 2 . This means that the orbit $\operatorname{Orb}\left(\widehat{\mathbf{x}}_{0}, \mathbb{S}_{\widehat{\alpha}}\right)$ is dense on the torus $\mathbb{T}^{2}$. Now our statement follows from Proposition 5.1 .

For nonmaximally irrational torus shifts $\mathbb{S}_{\widehat{\alpha}}$ the conclusion of Proposition 5.2 is false in general. This is easily seen if we note that by (5.15) the closure of the orbit $\overline{\operatorname{Orb}}\left(\widehat{\mathbf{x}}_{0}, \mathbb{S}_{\widehat{\alpha}}\right)$ is a one-dimensional winding of the torus $\mathbb{T}^{2}$ when $\operatorname{rank}_{\mathbb{Z}}(\widehat{\alpha})=1$, and a finite set of points when $\operatorname{rank}_{\mathbb{Z}}(\widehat{\alpha})=0$.

It follows that, instead of the boundary values $m(\widehat{T})$ and $M(\widehat{T})$ defined in (5.7), it is more natural to consider the new boundary values

$$
m\left(\widehat{x}_{0}, S_{\widehat{v}}\right)=\inf _{\widehat{x} \in \operatorname{Orb}\left(\widehat{x}_{0}, S_{\widehat{v}}\right)} \operatorname{pr}(\widehat{x}), \quad M\left(\widehat{x}_{0}, S_{\widehat{v}}\right)=\sup _{\widehat{x} \in \operatorname{Orb}\left(\widehat{x}_{0}, S_{\widehat{v}}\right)} \operatorname{pr}(\widehat{x}) .
$$

Since $\operatorname{Orb}\left(\widehat{x}_{0}, S_{\widehat{v}}\right) \subset \widehat{T}$, the boundary values in (5.7) and (5.16) satisfy the obvious inequalities

$$
m(\widehat{T}) \leq m\left(\widehat{x}_{0}, S_{\widehat{v}}\right), \quad M\left(\widehat{x}_{0}, S_{\widehat{v}}\right) \leq M(\widehat{T})
$$


Proposition 5.3. Suppose that for an exchange transformation $\mathbb{S}_{\widehat{v}}=\mathbb{S}_{\widehat{\alpha}}$ we have $\operatorname{rank}_{\mathbb{Z}}(\widehat{\alpha}) \leq 1$. Then for any coloring $\widehat{W}=\widehat{W}_{0} \sqcup \widehat{W}_{1}$ of the patch (1.12), the deviations $\delta_{k}\left(i, \widehat{\mathbf{x}}_{0}\right), k=0,1$, satisfy the sharp inequalities

$$
\begin{aligned}
& m\left(\widehat{x}_{0}, S_{\widehat{v}}\right)-x_{0} \leq \delta_{0}\left(i, \widehat{\mathbf{x}}_{0}\right) \leq M\left(\widehat{x}_{0}, S_{\widehat{v}}\right)-x_{0}, \\
& x_{0}-M\left(\widehat{x}_{0}, S_{\widehat{v}}\right) \leq \delta_{1}\left(i, \widehat{\mathbf{x}}_{0}\right) \leq x_{0}-m\left(\widehat{x}_{0}, S_{\widehat{v}}\right)
\end{aligned}
$$

for all $i=0,1,2, \ldots$

Proof. This follows immediately from formulas (5.4) for the deviations $\delta_{k}\left(i, \widehat{\mathbf{x}}_{0}\right)$ and from the definition (5.16) of the boundary values $m\left(\widehat{x}_{0}, S_{\widehat{v}}\right), M\left(\widehat{x}_{0}, S_{\widehat{v}}\right)$.

Remark 5.1. If $\operatorname{rank}_{\mathbb{Z}}(\widehat{\alpha})=2$, then inequalities (5.18) proved above give us inequalities (5.6) of Theorem 5.1.

5.5. Deviations for exchange transformations of the torus. Note that inequalities (5.18) are also valid for arbitrary exchange transformations $\mathbb{S}_{\widehat{v}}$ of the torus $\mathbb{T}^{2}$. A drawback of these inequalities is that to apply them one needs to know the structure of the orbit closures $\overline{\operatorname{Orb}}\left(\widehat{x}_{0}, S_{\widehat{v}}\right)$, which is a hard problem, solved to date only for some special cases of torus exchange transformations (see [2, 1]). Thus, the proof of sharp inequalities of the form (5.6) for the deviations $\delta_{k}\left(i, \widehat{\mathbf{x}}_{0}\right), k=0,1$, in the case of general exchange transformations $\mathbb{S}_{\widehat{v}}$ of the torus $\mathbb{T}^{2}$ is still an open problem.

\section{$\S 6$. EXCHANGE TRANSFORMATION OF FOUR INTERVALS:}

\section{A NUMERICAL EXAMPLE}

6.1. Torus development and its quotient. Let us apply Theorem 5.1 to the case where the fiber $T^{\prime}=[0,1)$ is partitioned,

$$
T^{\prime}=T_{0}^{\prime} \sqcup T_{1}^{\prime} \sqcup T_{2}^{\prime} \sqcup T_{3}^{\prime},
$$

into four different half-closed intervals $T_{k^{\prime}}^{\prime}=\left[\omega_{k^{\prime}}^{\prime}, \omega_{k^{\prime}+1}^{\prime}\right)$ with boundary points

$$
\begin{array}{cl}
w_{0}^{\prime}=0, & w_{1}^{\prime}=\sqrt{5}-2 \approx 0.24, \quad w_{2}^{\prime}=\sqrt{6}-2 \approx 0.45, \\
w_{3}^{\prime}=\sqrt{3}-1 \approx 0.73, \quad w_{4}^{\prime}=1 .
\end{array}
$$

Let an exchange transformation $S_{v^{\prime}}$ of the half-closed intervals $T_{k^{\prime}}^{\prime}$ be given by the per-

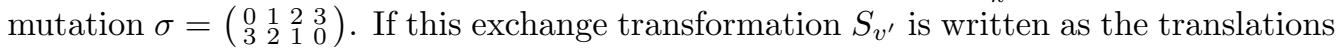

$$
S_{v^{\prime}}\left(x^{\prime}\right)=x^{\prime}+v_{k^{\prime}}^{\prime} \text { for } x^{\prime} \in T_{k^{\prime}}^{\prime},
$$

then the vectors $v_{k^{\prime}}^{\prime}$ will be, respectively,

$$
\begin{aligned}
& v_{0}^{\prime}=3-\sqrt{5} \approx 0.76, \quad v_{1}^{\prime}=5-\sqrt{5}-\sqrt{6} \approx 0.31, \\
& v_{2}^{\prime}=4-\sqrt{3}-\sqrt{6} \approx-0.18, \quad v_{3}^{\prime}=1-\sqrt{3} \approx-0.73 .
\end{aligned}
$$

Consider the partition $R=R_{0} \sqcup R_{1}$ of the real line $\mathbb{R}$ into two half-closed intervals $R_{0}=[-\infty, \omega), R_{1}=[\omega,+\infty$,$) with the cutting point \omega=1-\alpha \approx 0.59$, where $\alpha=\sqrt{2}-1$. Using this partition, we define a map $S_{v}$ on the base $\mathbb{R} \stackrel{S_{v}}{\longrightarrow} \mathbb{R}$ by setting (see (1.3) )

$$
S_{v}(x)=x+v_{k} \text { for } x \in R_{k},
$$

where

$$
v_{0}=\alpha=\sqrt{2}-1 \approx 0.41, \quad v_{1}=\alpha-1=\sqrt{2}-2 \approx-0.59 .
$$

We use a rectangle $\widehat{W}=W \times T^{\prime}$ with the base $W=\left[w_{0}, w_{1}\right)$ as a patch, where

$$
w_{0}=\omega+d_{0}, \quad w_{1}=\omega+d_{1} .
$$


The numbers $d_{0} \leq 0$ and $d_{1} \geq 0$ are called perturbations. In case where $d_{0}=0$ and $d_{1}=0$, the strip $\widehat{\mathbb{R}}$ has a partition $\widehat{\mathbb{R}}=\widehat{\mathbb{R}}_{0} \sqcup \widehat{\mathbb{R}}_{1}$ into two halves: the points $\widehat{x} \in \widehat{\mathbb{R}}_{0}$ with $x<\omega$ have color $\operatorname{col}(\widehat{x})=0$, while the points $\widehat{x} \in \widehat{\mathbb{R}}_{1}$ with $x \geq \omega$ have $\operatorname{color} \operatorname{col}(\widehat{x})=1$. If, on the other hand, $d_{0}-d_{1}>0$, then this partition is perturbed (see (1.13) ) by an arbitrary coloring of the patch $\widehat{W}$ with width

$$
|\widehat{W}|=|W|=d_{1}-d_{0} .
$$

As an addendum to Remark 6.1, now we present another argument in favor of the term 'perturbations' for the numbers $d_{0}, d_{1}$.

Next we choose $d_{0}=-0.1$ and $d_{1}=0.2$, obtaining the following values for the endpoints of the half-closed interval $W$ :

$$
w_{0}=\omega-0.1 \approx 0.49, \quad w_{1}=\omega+0.2 \approx 0.79 .
$$

Define a coloring on $\widehat{W}$ as follows:

$$
\widehat{W}=\widehat{W}_{0} \sqcup \widehat{W}_{1} \text {, where } \widehat{W}_{0}=W \times[0,0.5), \quad \widehat{W}_{1}=W \times[0.5,1) .
$$

In accordance with (2.3) and (2.4), we define a partition

$$
\widehat{R}=\coprod_{k, k^{\prime}} \widehat{R}_{k, k^{\prime}}
$$

of the strip $\widehat{\mathbb{R}}=\mathbb{R} \times T^{\prime}$ into eight regions $\widehat{R}_{k, k^{\prime}}$ with $k=0,1$ and $k^{\prime}=0,1,2,3$. Using the coloring (2.4), we define a translation map on the strip $S_{\widehat{v}}: \widehat{\mathbb{R}} \longrightarrow \widehat{\mathbb{R}}$ as in (2.6):

$$
S_{\widehat{v}}(\widehat{x})=\widehat{x}+\widehat{v}_{k, k^{\prime}} \text { for } \widehat{x} \in \widehat{R}_{k, k^{\prime}}
$$

where the shift vectors $\widehat{v}_{k, k^{\prime}}=\left(v_{k}, v_{k^{\prime}}^{\prime}\right)$ have coordinates defined in (6.3) and (6.4).

By Theorem 2.1, in order to construct a stabilizer $\widehat{T}$ of the map $S_{\widehat{v}}$ by using (2.7), we need to check condition (2.11). The width of the patch is calculated by (6.7), and $\min \left\{\left|v_{0}\right|,\left|v_{1}\right|\right\}=\left|v_{0}\right|=\sqrt{2}-1$ by (6.5). Therefore, condition (2.11) can be rewritten as

$$
d_{1}-d_{0}<\sqrt{2}-1 \approx 0.41
$$

The values $d_{0}=-0.1$ and $d_{1}=0.2$ chosen earlier satisfy inequality (6.10); hence, we may apply (2.7) to construct a stabilizer $\widehat{T}$ and define its partition

$$
\widehat{T}=\coprod_{k, k^{\prime}} \widehat{T}_{k, k^{\prime}}
$$

by using (2.10). Since the conditions of Theorem 2.1 are satisfied, we see that the set (6.11) is closed under the map $S_{\widehat{v}}$ :

$$
S_{\widehat{v}}: \widehat{T} \longrightarrow \widehat{T},
$$

and moreover, it is a development $\widehat{T}=T^{2}$ of the torus $\mathbb{T}^{2}$ with respect to the square lattice $L=\mathbb{Z}^{2}$. Figure 6.1 shows the stabilizer $\widehat{T}$ and its partition into eight parts $\widehat{T}_{k, k^{\prime}}$ (see (6.11) ), and Figure 6.2 shows the quotient $\mathbb{T}^{2} \simeq \widehat{T} \bmod \mathbb{Z}^{2}$ of the stabilizer $\widehat{T}$ with respect to the lattice $\mathbb{Z}^{2}$. The torus $\mathbb{T}^{2}$ is partitioned,

$$
\mathbb{T}^{2}=\mathbb{T}_{0}^{2} \sqcup \mathbb{T}_{1}^{2},
$$

into two regions $\mathbb{T}_{0}^{2}$ and $\mathbb{T}_{1}^{2}$, in accordance with (5.1). These regions correspond to the partition of the torus development

$$
\widehat{T}=\widehat{T}_{0} \sqcup \widehat{T}_{1}
$$




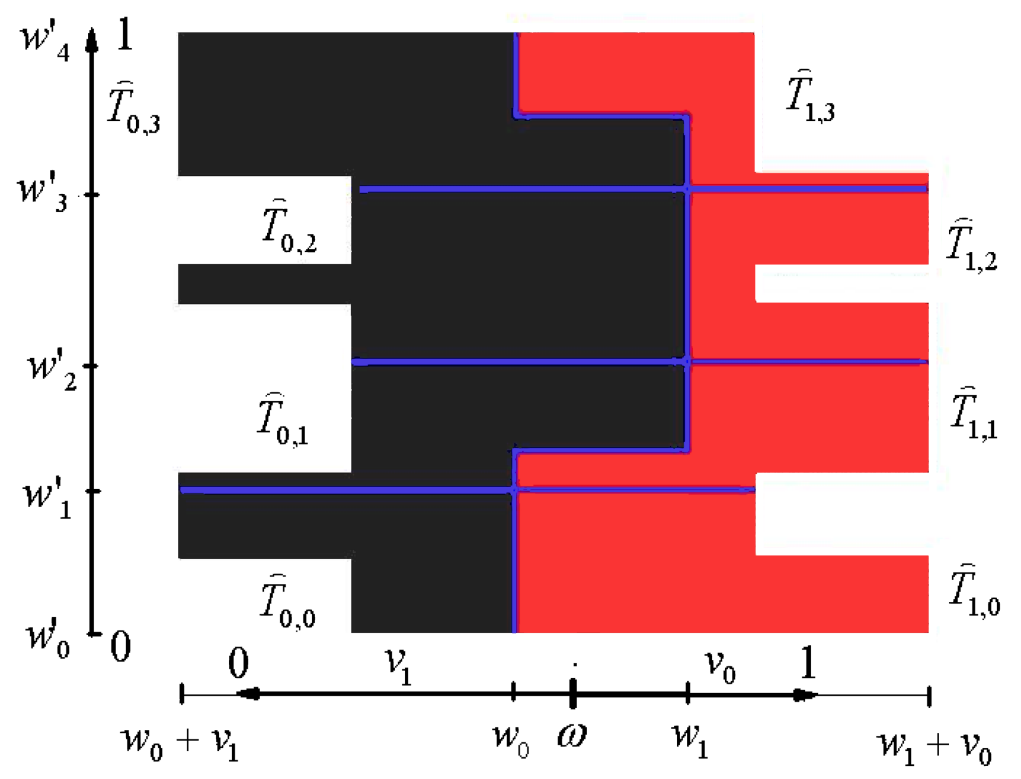

FiguRE 6.1. The stabilizer $\widehat{T}$ of the translation map $S_{\widehat{v}}$.

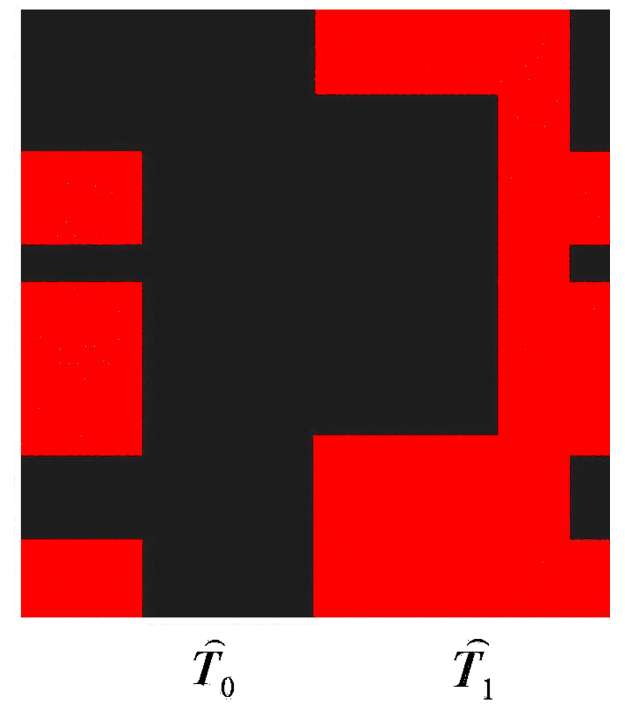

Figure 6.2. The quotient $\mathbb{T}^{2}$ of the torus development $\widehat{T} \bmod \mathbb{Z}^{2}$.

into the corresponding regions

$$
\begin{aligned}
& \widehat{T}_{0}=\widehat{T}_{0,0} \sqcup \widehat{T}_{0,1} \sqcup \widehat{T}_{0,2} \sqcup \widehat{T}_{0,3}, \\
& \widehat{T}_{1}=\widehat{T}_{1,0} \sqcup \widehat{T}_{1,1} \sqcup \widehat{T}_{1,2} \sqcup \widehat{T}_{1,3},
\end{aligned}
$$

where the sets $\widehat{T}_{k, k^{\prime}}$ are the same as in (6.11).

6.2. Incompressible colorings of the patch. Now we step aside of the main story and give several definitions. A coloring of the patch $\widehat{W}=\widehat{W}_{0} \sqcup \widehat{W}_{1}$ is said to be right (left) 
incompressible if for any $\varepsilon>0$ the strip $\widehat{W} \backslash \widehat{W}_{\varepsilon} \quad\left(\widehat{W} \backslash{ }_{\varepsilon} \widehat{W}\right)$, where $\widehat{W}_{\varepsilon}=\left[w_{0}, w_{1}-\varepsilon\right) \times \widehat{T}$ and ${ }_{\varepsilon} \widehat{W}=\left[w_{0}+\varepsilon, w_{1}\right) \times \widehat{T}$, contains at least one point $\widehat{x}$ with $\operatorname{col}(\widehat{x})=0 \quad(\operatorname{col}(\widehat{x})=1)$. A coloring is incompressible if it is both right incompressible and left incompressible. For example, the coloring in (6.9) of the patch $\widehat{W}$ is incompressible (see Figure 6.1).

Lemma 6.1. If a coloring of the patch $\widehat{W}=\widehat{W}_{0} \sqcup \widehat{W}_{1}$ is incompressible, and the perturbations $d_{0} \leq 0$ and $d_{1} \geq 0$ (see (․6.6) $)$ satisfy

$$
d_{1}-d_{0}<\min \left\{\left|v_{0}\right|,\left|v_{1}\right|\right\}
$$

then the following statements are true.

1. The set $\widehat{T}$ is closed under the map $S_{\widehat{v}}$, and it is a development $\widehat{T}=T^{2}$ of the torus $\mathbb{T}^{2}$ with respect to the square lattice $L=\mathbb{Z}^{2}$.

2. The boundary values $m(\widehat{T})$ and $M(\widehat{T})$ defined in (5.7) are

$$
m(\widehat{T})=d_{0}, \quad M(\widehat{T})=1+d_{1} .
$$

Proof. The first statement follows from Theorem 2.1] because inequality (6.15) is equivalent to (2.11).

In order to prove the second statement, observe that the construction in (2.7) of the torus development $\widehat{T}$ together with the incompressibility of the coloring of the patch $\widehat{W}=\widehat{W}_{0} \sqcup \widehat{W}_{1}$ implies that for the boundary values $m(\widehat{T})$ and $M(\widehat{T})$ we have

$$
m(\widehat{T})=w_{0}+v_{1}, \quad M(\widehat{T})=w_{1}+v_{0} .
$$

Substituting in (6.17) the values of $v_{0}, v_{1}$ and $w_{0}, w_{1}$ from (6.5) and (6.6), we obtain (6.16).

Now we can specify estimates (5.6) of Theorem 5.1 in the case of incompressible colorings of the patch $\widehat{W}$.

Proposition 6.1. Let $\widehat{\mathbf{x}}_{0}$ be an arbitrary fixed starting point on the torus $\mathbb{T}^{2}$, and let $\mathbb{S}_{\widehat{v}}$ be an exchange transformation of the torus corresponding to the map $S_{\widehat{v}}$ via (3.4). Suppose that the conditions of Lemma 6.1 are fulfilled. Then the following statements are true.

1. The deviation functions $\delta_{k}\left(i, \widehat{\mathbf{x}}_{0}\right), k=0,1$, defined in (5.3) satisfy the inequalities

$$
\begin{aligned}
d_{0}-x_{0} & \leq \delta_{0}\left(i, \widehat{\mathbf{x}}_{0}\right) \leq 1+d_{1}-x_{0}, \\
x_{0}-1-d_{1} \leq \delta_{1}\left(i, \widehat{\mathbf{x}}_{0}\right) & \leq x_{0}-d_{0}
\end{aligned}
$$

for all $i=0,1,2, \ldots$ Here $x_{0}=\operatorname{pr}\left(\widehat{\mathbf{x}}_{0}\right)$ is the projection (3.25) of the point $\widehat{\mathbf{x}}_{0}$ to the line $\mathbb{R}$, and $d_{0} \leq 0, d_{1} \geq 0$ are the perturbations, see (6.6).

2. For an arbitrary starting point $\widehat{\mathbf{x}}_{0}$ on the torus $\mathbb{T}^{2}$, the general inequalities

$$
\left|\delta_{0}\left(i, \widehat{\mathbf{x}}_{0}\right)\right| \leq 1+|\widehat{W}|, \quad\left|\delta_{1}\left(i, \widehat{\mathbf{x}}_{0}\right)\right| \leq 1+|\widehat{W}|
$$

are valid for all $i=0,1,2, \ldots$, where $|\widehat{W}|=d_{1}-d_{0}$ is the width of the patch $\widehat{W}$.

Remark 6.1. The name 'perturbations' for $d_{0}$ and $d_{1}$, given in (6.6), is explained by inequalities (6.18) and (6.19): as these numbers grow, the bounds for the deviation functions $\delta_{0}\left(i, \widehat{\mathbf{x}}_{0}\right), \delta_{1}\left(i, \widehat{\mathbf{x}}_{0}\right)$ also grow.

Proof of Proposition 6.1. 1. Inequalities (6.18) follow from Theorem 5.1 and Lemma 6.1

2. Using the bijection $\widehat{\iota}$ defined in (3.14) , we obtain a point $\widehat{x}_{0}=\left(x_{0}, x_{0}^{\prime}\right)=\widehat{\iota}^{-1}\left(\widehat{\mathbf{x}}_{0}\right)$. Since it lies in the torus development $\widehat{T}$, Lemma 6.1 says that the abscissa $x_{0}$ of the point $\widehat{x}_{0}$ satisfies $d_{0} \leq x_{0} \leq 1+d_{1}$. Together with (6.18), this gives us (6.19). 
6.3. Bounds for deviation functions. Now we return to the transformation map $S_{\widehat{v}}$ defined in (6.12). Let

$$
\delta_{0}\left(i, \widehat{\mathbf{x}}_{0}\right)=\mathbf{r}_{0}\left(i, \widehat{\mathbf{x}}_{0}\right)-i a_{0}, \quad \delta_{1}\left(i, \widehat{\mathbf{x}}_{0}\right)=\mathbf{r}_{1}\left(i, \widehat{\mathbf{x}}_{0}\right)-i a_{1}
$$

be the deviation functions (5.3), where $\mathbf{r}_{0}\left(i, \widehat{\mathbf{x}}_{0}\right)$ and $\mathbf{r}_{1}\left(i, \widehat{\mathbf{x}}_{0}\right)$ are the distribution functions (5.2) for points of the orbits $\operatorname{Orb}\left(\widehat{\mathbf{x}}_{0}, \mathbb{S}_{\widehat{v}}\right)$ over the regions $\mathbb{T}_{0}^{2}, \mathbb{T}_{1}^{2}$ in the torus $\mathbb{T}^{2}$. In our case, the coefficients $a_{0}, a_{1}$ from (6.20) look like this:

$$
a_{0}=1-\alpha=2-\sqrt{2} \approx 0.59, \quad a_{1}=\alpha=\sqrt{2}-1 \approx 0.41,
$$

and these coefficients are equal to the areas $\operatorname{vol}\left(\mathbb{T}_{0}^{2}\right)$ and $\operatorname{vol}\left(\mathbb{T}_{1}^{2}\right)$ of the regions $\mathbb{T}_{0}^{2}$ and $\mathbb{T}_{1}^{2}$, shown in Figure 6.2. Note that the entire torus $\mathbb{T}^{2}$ has area $\operatorname{vol}\left(\mathbb{T}^{2}\right)=1$.

As was noted before, the coloring of the patch $\widehat{W}$ defined in (6.9) is incompressible. Moreover, by (6.10), condition (6.15) of Lemma 6.1 is fulfilled. Hence, we can apply Proposition 6.1 to our exchange transformation map $S_{\widehat{v}}$. Recall that $d_{0}=-0.1, d_{1}=0.2$. Therefore, the width of the patch is $|\widehat{W}|=d_{1}-d_{0}=0.3$, and we have two-sided inequalities for the deviations in (6.20),

$$
\begin{aligned}
-0.1-x_{0} & \leq \delta_{0}\left(i, \widehat{\mathbf{x}}_{0}\right) \leq 1.2-x_{0}, \\
x_{0}-1.2 & \leq \delta_{1}\left(i, \widehat{\mathbf{x}}_{0}\right) \leq x_{0}+0.1,
\end{aligned}
$$

and the general inequalities

$$
\left|\delta_{0}\left(i, \widehat{\mathbf{x}}_{0}\right)\right| \leq 1.3, \quad\left|\delta_{1}\left(i, \widehat{\mathbf{x}}_{0}\right)\right| \leq 1.3
$$

for any starting point $\widehat{\mathbf{x}}_{0}$ on the torus $\mathbb{T}^{2}$ and for all $i=0,1,2, \ldots$

\section{$\S 7$. Mean values of the Deviation function}

7.1. $\mu$-admissible exchange transformations of torus developments. For the deviation function $\delta_{k}\left(i, \widehat{\mathbf{x}}_{0}\right)$ introduced in (5.3), we define its mean value by

$$
\left\langle\delta_{k}\left(\widehat{\mathbf{x}}_{0}\right)\right\rangle=\lim _{N \rightarrow+\infty} \frac{1}{N} \sum_{0 \leq i \leq N-1} \delta_{k}\left(i, \widehat{\mathbf{x}}_{0}\right)
$$

if the limit in (7.1) exists.

Assume that $k=0$. Substituting $\delta_{0}\left(i, \widehat{\mathbf{x}}_{0}\right)=x_{i}-x_{0}$, we obtain

$$
\lim _{N \rightarrow+\infty} \frac{1}{N} \sum_{0 \leq i \leq N-1} \delta_{0}\left(i, \widehat{\mathbf{x}}_{0}\right)=\lim _{N \rightarrow+\infty} \frac{1}{N} \sum_{0 \leq i \leq N-1} \operatorname{pr}\left(S_{\widehat{v}}^{i}\left(\widehat{x}_{0}\right)\right)-x_{0} .
$$

On the right-hand side of (7.2) we have replaced $x_{i}$ by $x_{i}=\operatorname{pr}\left(\mathbb{S}_{\widehat{v}}^{i}\left(\widehat{\mathbf{x}}_{0}\right)\right)=\operatorname{pr}\left(S_{\widehat{v}}^{i}\left(\widehat{x}_{0}\right)\right)$, see (5.5).

Next we wish to replace the limit value of the sum on the right-hand side of (7.2) with a certain integral over the torus development $\widehat{T}$. This procedure is well known for strictly ergodic transformations (homeomorphisms $S$ of compact metric spaces $X$ with a unique invariant Borel measure $\mu$, see the survey [17]). For exchange transformations of multidimensional domains, minimality is known only in special cases. The author is not aware of any results on their strict ergodicity. Below we introduce two axiomatic notions which imply the ergodicity properties for the transformations $S_{\widehat{v}}$ that we are interested in. Another reason to bypass the ergodic theorems temporarily is the structure of the development $\widehat{T}$ itself, which may not even be integrable.

We say that the exchange transformation map of the development $S_{\widehat{v}}: \widehat{T} \longrightarrow \widehat{T}$ in Theorem 2.1 is $\mu$-admissible if there exists a measure $\mu(\widehat{x})$ on $\widehat{T}$ such that the integral 
$\int_{\widehat{T}} x d \mu(\widehat{x})$ exists, and for any starting point $\widehat{x}_{0} \in \widehat{T}$ we have

$$
\lim _{N \rightarrow+\infty} \frac{1}{N} \sum_{0 \leq i \leq N-1} \operatorname{pr}\left(S_{\widehat{v}}^{i}\left(\widehat{x}_{0}\right)\right)=\int_{\widehat{T}} x d \mu(\widehat{x}),
$$

where $x$ is the coordinate of a point $\widehat{x}=\left(x, x^{\prime}\right)$ in $\widehat{T}$. In what follows, the measure $\mu(\widehat{x})$ is assumed to be normalized, i.e., $\int_{\widehat{T}} d \mu(\widehat{x})=1$. The existence of the integral in (7.3) depends on the coloring of the strip $\widehat{W}=\widehat{W}_{0} \sqcup \widehat{W}_{1}$ in (2.11). A coloring is said to be integrable with respect to the measure $\mu(\widehat{x})$ if the integrals below exist and satisfy

$$
\int_{\widehat{W}_{0}} x d \mu(\widehat{x})+\int_{\widehat{W}_{1}} x d \mu(\widehat{x})=\int_{\widehat{W}} x d \mu(\widehat{x}) .
$$

The construction (2.7) of the torus development $\widehat{T}$ and the definition (7.4) of the integrability of the strip coloring $\widehat{W}=\widehat{W}_{0} \sqcup \widehat{W}_{1}$ imply the existence of the integral on the right-hand side of (17.3).

Theorem 7.1. Assume that there exists a measure $\mu$ on the torus development $\widehat{T}$ such that the exchange transformation map $S_{\widehat{v}}: \widehat{T} \longrightarrow \widehat{T}$ defined in (2.13) is $\mu$-admissible, see (7.3). Then for any starting point $\widehat{\mathbf{x}}_{0}$ on $\mathbb{T}^{2}$ the mean values $\left\langle\delta_{k}\left(\widehat{\mathbf{x}}_{0}\right)\right\rangle$ for the deviation function $\delta_{k}\left(i, \widehat{\mathbf{x}}_{0}\right)$ defined in (7.1) exist, and

$$
\left\langle\delta_{0}\left(\widehat{\mathbf{x}}_{0}\right)\right\rangle=\int_{\widehat{T}} x d \mu(\widehat{x})-x_{0}, \quad\left\langle\delta_{1}\left(\widehat{\mathbf{x}}_{0}\right)\right\rangle=x_{0}-\int_{\widehat{T}} x d \mu(\widehat{x}),
$$

where $x_{0}=\operatorname{pr}\left(\widehat{\mathbf{x}}_{0}\right)$ is the projection (3.25) of the starting point $\widehat{\mathbf{x}}_{0} \in \mathbb{T}^{2}$ to the real line $\mathbb{R}$.

Proof. The first formula in (7.5) follows from the definition (7.1) of the mean value $\left\langle\delta_{k}\left(\widehat{\mathbf{x}}_{0}\right)\right\rangle$ and from formulas (7.2), (7.3). In order to prove the second part of (7.5), observe that, by (5.4), the deviations $\delta_{0}\left(i, \widehat{\mathbf{x}}_{0}\right)$ and $\delta_{1}\left(i, \widehat{\mathbf{x}}_{0}\right)$ satisfy $\delta_{1}\left(i, \widehat{\mathbf{x}}_{0}\right)=-\delta_{0}\left(i, \widehat{\mathbf{x}}_{0}\right)$, which implies the corresponding relation for the mean values: $\left\langle\delta_{1}\left(\widehat{\mathbf{x}}_{0}\right)\right\rangle=-\left\langle\delta_{0}\left(\widehat{\mathbf{x}}_{0}\right)\right\rangle$.

7.2. Mean values of deviations for torus shifts. Suppose that an exchange transformation map $\mathbb{S}_{\widehat{v}}=\mathbb{S}_{\widehat{\alpha}}$ is the shift $\mathbb{S}_{\widehat{\alpha}}(\widehat{x}) \equiv \widehat{x}+\widehat{\alpha} \bmod \mathbb{Z}^{2}$ of the torus $\mathbb{T}^{2}$ by a vector $\widehat{\alpha}=\left(\alpha, \alpha^{\prime}\right)$. Assume that the vector $\widehat{\alpha}$ is maximally irrational (see Subsection 5.4 ). We take the standard volume measure (or Lebesgue measure) for the role of a measure $\mu(\widehat{x})$ on the torus development $\widehat{T} \subset \mathbb{R}^{2}$. In this case we have

$$
d \mu(\widehat{x})=d x d x^{\prime}
$$

where $x, x^{\prime}$ are coordinates of a point $\widehat{x}=\left(x, x^{\prime}\right)$ on the plane $\mathbb{R}^{2}$.

For the torus shift $\mathbb{S}_{\widehat{\alpha}}$ to be $\mu$-admissible for the measure (7.6), we need the integrability of the patch coloring $\widehat{W}=\widehat{W}_{0} \sqcup \widehat{W}_{1}$ from (1.12), i.e., we need the formula

$$
\int_{\widehat{W}_{0}} x d x d x^{\prime}+\int_{\widehat{W}_{1}} x d x d x^{\prime}=\int_{\widehat{W}} x d x d x^{\prime},
$$

where the integrals on the left-hand side are assumed to exist.

Proposition 7.1. Assume that the shift $\mathbb{S}_{\widehat{\alpha}}$ of the torus $\mathbb{T}^{2}$ is maximally irrational, and that the patch coloring $\widehat{W}=\widehat{W}_{0} \sqcup \widehat{W}_{1}$ from (1.12) satisfies condition (7.7). Then for any starting point $\widehat{\mathbf{x}}_{0}$ in $\mathbb{T}^{2}$ the mean values $\left\langle\delta_{k}\left(\widehat{\mathbf{x}}_{0}\right)\right\rangle$, as defined in (7.1), exist and satisfy

$$
\left\langle\delta_{0}\left(\widehat{\mathbf{x}}_{0}\right)\right\rangle=\int_{\widehat{T}} x d x d x^{\prime}-x_{0}, \quad\left\langle\delta_{1}\left(\widehat{\mathbf{x}}_{0}\right)\right\rangle=x_{0}-\int_{\widehat{T}} x d x d x^{\prime},
$$

where $x_{0}=\operatorname{pr}\left(\widehat{\mathbf{x}}_{0}\right)$ is the projection of the starting point $\widehat{\mathbf{x}}_{0} \in \mathbb{T}^{2}$ to the line $\mathbb{R}$. 
Proof. The existence of the integral $\int_{\widehat{T}} x d \mu(\widehat{x})$ follows from condition (7.7) and the construction (2.7) of the torus development $\widehat{T}$. Combined with the maximal irrationality of the torus shift $\mathbb{S}_{\widehat{\alpha}}$, this implies formula (7.3), which together with (7.1), (7.2) implies (7.8).

Remark 7.1. Geometric considerations show that the value of the integral

$$
c_{\widehat{T}, x}=\int_{\widehat{T}} x d x d x^{\prime}
$$

occurring in (7.8) is simply the projection $c_{\widehat{T}, x}=\operatorname{pr}\left(c_{\widehat{T}}\right)$ of the center of mass $c_{\widehat{T}}$ of the torus development $\widehat{T}$ to the line $\mathbb{R}$.

Remark 7.2. Formulas (7.8) remain valid for nonmaximally irrational shifts $\mathbb{S}_{\widehat{\alpha}}$, when for the rank of the shift vector we have $\operatorname{rank}_{\mathbb{Z}}(\widehat{\alpha}) \leq 1$. We only need to return to the general formulas of Theorem [7.1, rewriting them as

$$
\left\langle\delta_{0}\left(\widehat{\mathbf{x}}_{0}\right)\right\rangle=\int_{\widehat{O}} x d \mu(\widehat{x})-x_{0}, \quad\left\langle\delta_{1}\left(\widehat{\mathbf{x}}_{0}\right)\right\rangle=x_{0}-\int_{\widehat{O}} x d \mu(\widehat{x}) .
$$

In these integrals, the torus development $\widehat{T}$ is replaced with $\widehat{O}=\overline{\mathrm{Orb}}\left(\widehat{x}_{0}, S_{\widehat{v}}\right)$, the closure of the orbit $\operatorname{Orb}\left(\widehat{x}_{0}, S_{\widehat{v}}\right)$. The measure $\mu(\widehat{x})$ in the integrals in (7.10) should be replaced by the normalized length measure in the case where $\operatorname{rank}_{\mathbb{Z}}(\widehat{\alpha})=1$, and by the normalized discrete measure in the case where $\operatorname{rank}_{\mathbb{Z}}(\widehat{\alpha})=0$. As in (17.9), the values $c_{\widehat{O}, x}$ of the integrals in (7.10) can then be interpreted as the projection of the center of mass $\widehat{O}$ to the line $\mathbb{R}$.

\section{REFERENCES}

[1] J. Athreya and M. Boshernitzan, Ergodic properties of compositions of interval exchange maps and rotations, Nonlinearity 26 (2013), no. 2, 417-423. MR3007897

[2] H. Haller, Rectangle exchange transformations, Monatsh. Math. 91 (1981), no. 3, $215-232$. MR619965 (83b:28019)

[3] E. Hecke, Uber analytische Funktionen und die Verteilung von Zahlen mod. eins, Abh. Math. Sem. Univ. Hamburg. 1 (1921), no. 1, 54-76. MR.3069388

[4] S. Ferenczi, Bounded remainder sets, Acta Arith. 61 (1992), no. 4, 319-326. MR 1168091 (93f:11059)

[5] P. Liardet, Regularities of distribution, Compositio Math. 61 (1987), no. 3, 267-293. MR883484 (88h:11052)

[6] I. Oren, Admissible functions with multiple discontinuities, Proc. Special Sem. Topology, Vol. 1 (Mexico City, 1980/1981), Univ. Nac. Autónoma México, Mexico City, 1981, pp. 217-230. MR658174 (83j:54034)

[7] G. Rauzy, Ensembles à restes bornés, Sem. Number Theory, 1983-1984 (Talence, 1983/1984), Exp. No. 24, Univ. Bordeaux, Talence, 1984. MR784071 (86g:28024)

[8] P. Szüsz, Über die Verteilung der Vielfachen einer komplexen Zahl nach dem Modul des Einheitsquadrats, Acta Math. Acad. Sci. Hungar. 5 (1954), 35-39. MR0064086 (16:224a)

[9] H. Weyl, Über die Gleichverteilung von Zahlen mod Eins, Math. Ann. 77 (1916), no. 3, 313-352. MR 1511862

[10] G. F. Voronoi, Collected works. Vol. II, Izdat. Akad. Nauk USSR, Kiev, 1952. (Russian) MR0062686 $(16: 2 \mathrm{~d})$

[11] V. G. Zhuravlev, Rouzy tilings and bounded remainder sets on a torus, Zap. Nauchn. Sem. S.Peterburg. Otdel. Mat. Inst. Steklov. (POMI) 322 (2005), 83-106; English transl., J. Math. Sci. (N.Y.) 137 (2006), no. 2, 4658-4672. MR2138453 (2006b:11094)

[12] _ A multidimensional Hecke theorem on the distribution of fractional parts, Algebra $\mathrm{i}$ Analiz 24 (2012), no. 1, 95-130; English transl., St. Petersburg Math. J. 24 (2013), no. 1, 7197. MR3013295

[13] Exchanged toric developments and bounded remainder sets, Zap. Nauchn. Sem. S.Peterburg. Math. Inst. Steklov. (POMI) 392 (2011), 95-145; English transl., J. Math. Sci. (N.Y.) 184 (2012), no. 6, 716-745. MR2870222 
[14] - Moduli of toric tilings into bounded remainder sets and balanced words, Algebra i Analiz 24 (2012), no. 4, 97-136; English transl., St. Petersburg Math. J. 24 (2013), no. 4, 601-629. MR.3088009

[15] _ Bounded remainder polyhedra, Sovrem. Probl. Mat., vyp. 16, MIAN, Moscow, pp. 82-102; English transl., Proc. Steklov Inst. Math. 280 (2013), no. 2, 71-90.

[16] _ Multi-colour dynamical tilings of tors into bounded remainder sets, Izv. Ross. Akad. Nauk Ser. Mat. (to appear).

[17] V. V. Kozlov, Weighted averages, uniform distribution and strict ergodicity, Uspekhi Mat. Nauk 60 (2005), no. 6, 115-138; English transl., Russian Math. Surveys 60 (2005), no. 6, 1121-1146. MR2215757 (2007h:37005)

[18] E. S. Fedorov, Elements of the study of figures, Izdat. Akad. Nauk SSSR, Moscow, 1953. (Russian) MR0062061 (15:923q)

Vladimir State University, PR. Stroiteley 11, 600024 Vladimir, Russia

E-mail address: vzhuravlev@mail.ru

Received 1/SEP/2014

Translated by A. LUZGAREV 